\title{
Robert Gugutzer*
}

\section{Leib und Situation. Zum Theorie- und Forschungsprogramm der Neophänomenologischen Soziologie} Felt Body and Situation. To the Theory and Research Program of the Neophenomenological Sociology

DOI 10.1515/zfsoz-2017-1009

Zusammenfassung: Die phänomenologisch orientierte Soziologie steht überwiegend in der Tradition der Transzendentalphänomenologie Edmund Husserls und der Mundanphänomenologie Alfred Schütz'. Sie präsentiert sich daher vor allem als Lebenswelt-, Alltags- und Wissenssoziologie. Abgrenzend dazu skizziert der Beitrag eine alternative phänomenologische Soziologie, deren philosophisches Fundament die Neue Phänomenologie von Hermann Schmitz ist. Auf dieser Grundlage, allen voran der Leib- und Situationstheorie von Schmitz, werden die Grundzüge der Neophänomenologischen Soziologie (NPS) vorgestellt. Ihre wesentlichen Bausteine sind (1) das leiblich-affektive Betroffensein als präpersonales Apriori des Sozialen, (2) leibliche Kommunikation als Quelle der Du-Evidenz und kleinste Analyseeinheit des Sozialen sowie (3) die gemeinsame Situation als sozialontologisches Fundament und empirische Erscheinungsform von Sozialität.

Schlüsselwörter: Leib; Situation; Neue Phänomenologie; Hermann Schmitz; Sozialtheorie; transhumane Soziologie; methodologischer Situationismus.

\footnotetext{
Abstract: Phenomenologically oriented sociology stands predominantly in the tradition of the transcendental phenomenology of Edmund Husserl and the mundane phenomenology of Alfred Schütz. It therefore presents itself primarily as sociology of the lifeworld or everyday life, and as sociology of knowledge. In contrast, this article sketch-

*Korrespondenzautor: Robert Gugutzer, Goethe-Universität Frankfurt a. M., Institut für Sportwissenschaften, Abt. Sozialwissenschaften des Sports, Ginnheimer Landstraße 39, 60487 Frankfurt a. M., Tel.: 069/798-245 29, Fax: 069/798-245 39, E-mail: gugutzer@sport.uni-frankfurt.de
}

es an alternative phenomenological sociology, the philosophical foundation of which is the New Phenomenology of Hermann Schmitz. It is particularly the theory of the felt body (Leib) and the situation theory of Schmitz, which are presented as the basic elements of Neophenomenological Sociology (NPS). The article outlines especially three aspects of the theory and research program of the NPS: (1) The affective involvement as a pre-personal a priori of the social, (2) the felt-bodily (leibliche) communication as the source of "Du-Evidenz" and as smallest analytical unit of the social, as well as (3) the common situation as a social-ontological foundation and empirical manifestation of sociality.

Keywords: Felt Body; Situation; Neophenomenology; Hermann Schmitz; Social Theory; Trans-Human Sociology; Methodological Situationism.

\section{Einleitung}

Seit den grundlagentheoretischen Arbeiten von Alfred Schütz zählt die Phänomenologie zu den wichtigsten philosophischen Disziplinen innerhalb der Soziologie. Neben ihrer fundamentalen Bedeutung für die im Anschluss an Schütz so genannte phänomenologische Soziologie zeigt sich der Einfluss der Phänomenologie besonders in der Ethnomethodologie, der Wissenssoziologie und der Methodologie qualitativer Sozialforschung, aber auch in Luhmanns Systemtheorie, Habermas' Kritischer Theorie oder Giddens' Strukturationstheorie hat sie Eingang gefunden. Auffällig ist dabei, dass die soziologische Auseinandersetzung mit der phänomenologischen Philosophie auf einen engen Kreis an Autoren und Themen begrenzt ist. Seit 
den wegweisenden Untersuchungen von Schütz bildet die Transzendentalphänomenologie von Edmund Husserl den unumstrittenen Referenzpunkt für die Soziologie (vgl. Schütz 1960, 1971a, 1971b). Kernthemen der durch Husserl inspirierten phänomenologischen Soziologie sind die bewusstseinsmäßige Konstitution sinnhaften Handelns und die soziale, vor allem wissensbasiert-kommunikative Konstruktion gesellschaftlicher Wirklichkeit, die Problematik des Fremdverstehens und der Intersubjektivität sowie die Lebensweltthematik. In Fortführung der Husserl-SchützTradition präsentiert sich die phänomenologische Soziologie gegenwärtig vor allem als Lebenswelt-, Alltags- und Wissenssoziologie (vgl. die Übersichten in Bühl 2002; Eberle 2012; Fischer 2012; Knoblauch 2009; Raab et al. 2008; für den angloamerikanischen Raum siehe Bird 2009; Ferguson 2006; Nasu 2012).

Neben Husserls Transzendentalphänomenologie dürfte die Wahrnehmungsphänomenologie von Maurice Merleau-Ponty die von der Soziologie bevorzugteste phänomenologische Philosophie sein. Die Soziologie rekurriert auf Merleau-Ponty insbesondere dann, wenn das Verhältnis von Leiblichkeit/Sinnlichkeit und Intersubjektivität/Sozialität in Frage steht. Das soziologische Interesse gilt hierbei vor allem Merleau-Pontys Konzept der „Zwischenleiblichkeit“ (Merleau-Ponty 1994: 185), das vorzugsweise für eine Kritik an Schütz’ egologischem Ansatz und die Entwicklung einer Sozialitätstheorie genutzt wird, deren Fundament die fungierende Leiblichkeit ist: Der Leib als Medium des Handelns und als Vermittler zwischen Ich und Welt (vgl. exemplarisch Bongaerts 2003; Coenen 1985a, 1985b; Crossley 1995, 1996; Fischer 2003; Meyer-Drawe 1984; Taylor 1986). Des Weiteren finden Merleau-Pontys leibphänomenologische Überlegungen große Resonanz im Kontext der jüngeren Körpersoziologie, allen voran in den Diskussionen um eine körperbasierte, embodied sociology (als Überblick siehe Alkemeyer 2015; Gugutzer 2012, 2015a).

Verglichen mit dem zentralen Stellenwert der Phänomenologien von Husserl und Merleau-Ponty sind jene von Scheler, Heidegger, Sartre, Levians, Ricoeur oder Waldenfels wie auch die derzeit besonders populäre „enactive phenomenology“ von Gallagher soziologisch randständig. Nahezu außerhalb des Wahrnehmungsfeldes der Soziologie befindet sich schließlich die Neue Phänomenologie von Hermann Schmitz ( $\left.{ }^{\star} 1928\right)$. Schmitz’ Schriften $^{1}$ werden vom Großteil der phänomenologisch orien-

1 Schmitz' zentrales Werk ist sein aus fünf Bänden mit zehn Teilbänden bestehendes und mehr als 5000 Seiten umfassendes „System der Philosophie“, das er zwischen 1964 und 1980 vorgelegt hat (Schmitz 1964-1980b). Das Schlagwort „Neue Phänomenologie“ gebrauchte tierten Soziologinnen und Soziologen ignoriert. ${ }^{2}$ Über die Gründe für das Desinteresse an Schmitz' Philosophie kann nur spekuliert werden, aber vermutlich spielt eine wichtige Rolle, dass die Neue Phänomenologie wenig mit der alten Phänomenologie gemein hat. Schmitz’ Phänomenologie bricht nämlich mit einigen Grundannahmen der traditionellen Phänomenologie und widmet sich von dieser marginalisierten oder unentdeckten Themen. Dazu zählen insbesondere der Leib als das Sich-Spüren in der Gegend des eigenen Körpers, die leibliche Kommunikation als Grundform der Wahrnehmung, Subjektivität im Sinne subjektiver Tatsachen, Gefühle als räumlich ergossene Atmosphären, eine anti-reduktionistische Raumtheorie mit der Unterscheidung zwischen Weite-, Orts- und Richtungsraum, Situation als ontologischer Grundbegriff sowie Halbdinge als Phänomene zwischen Dingen und Sinnesdaten (vgl. als Überblick Schmitz 2016). Allein angesichts dieser thematischen Innovationen ist es in der Tat gerechtfertigt, von einer Neuen Phänomenologie zu sprechen.

Auf die Gemeinsamkeiten und Unterschiede zwischen alter und Neuer Phänomenologie kann an dieser Stelle nicht genauer eingegangen werden. Im Hinblick auf die weitere Argumentation seien lediglich zwei besonders wichtige Divergenzen hervorgehoben: Während Husserls Phänomenologie durch ein „Primat des Bewusstseins“ (Suber 2007: 477) sowie eine „triadische Dingontologie“ (Schmitz 2002: 15) und daher einem Denken in „Konstellation“ (Schmitz 2003: 372) gekennzeichnet ist, herrscht in Schmitz’ Phänomenologie das Primat des „affektiven Betroffenseins“ bzw. des Leibes und vertritt sie eine Situ-

Schmitz erstmals als Titel einer kleinen, 1980 erschienenen Publikation (Schmitz 1980c). Für eine komprimierte Zusammenfassung des „Systems“ vgl. Schmitz (1990), für einen Überblick über die zentralen Bausteine der Neuen Phänomenologie siehe Schmitz (2003, 2009). Sehr gute Einführungen in Schmitz' Werk bieten Lauterbach (2014: 415-516) und Soentgen (1998).

2 Zu den Ausnahmen zählt zu allererst Gesa Lindemann, die meines Wissens als erste Schmitz in die Soziologie eingeführt hat (vgl. Lindemann 1992, 1993, 1996). Stand zu Anfang Schmitz' Leibtheorie im Mittelpunkt von Lindemanns Arbeiten (siehe dazu auch Lindemann 2017), so nutzt sie mittlerweile ebenso dessen Raum- und Mannigfaltigkeitstheorie (vgl. Lindemann 2014). Auf Schmitz’ Leibphänomenologie rekurrieren ebenfalls Anke Abraham (2002), Fritz Böhle (Böhle \& Fross 2009, Böhle \& Porschen 2011) oder Ulle Jäger (2004), auf Schmitz' Normentheorie Schützeichel (2016). Die umfassendste und fundierteste soziologische Auseinandersetzung mit Schmitz' Werk stellt Michael Uzarewicz' (2011) Entwurf einer neophänomenologischen „Soziologie des Transhumanen“ dar. In meinen eigenen Arbeiten stand zuerst Schmitz' Leibphänomenologie im Mittelpunkt (Gugutzer 2002, 2004, 2005, 2008, 2010, 2012, 2014, 2015b), inzwischen auch seine Situationstheorie (Gugutzer 2015c, 2015d, 2016a, 2016b, 2017a). 
ationsontologie (s. Abschnitte 2 und 4). Die an Schmitz' Phänomenologie anschließende Neophänomenologische Soziologie (NPS), ${ }^{3}$ um deren Entwurf es hier gehen soll, teilt deren Grundannahmen und versteht sich daher im Kern als eine leib- und situationsbasierte Soziologie.

Die NPS ist bislang mehr ein Programm denn ein theoretisch und methodologisch umfassend ausgearbeiteter soziologischer Ansatz. Abgesehen von den Arbeiten von Uzarewicz (2011) und Gugutzer (2012) liegen keine Untersuchungen vor, die sich ernsthaft an der Entwicklung einer dezidiert neophänomenologischen Sozial- oder gar Gesellschaftstheorie versucht haben. Vor dem Hintergrund dieses Desiderats ist es das primäre Ziel des vorliegenden Beitrags, das Theorie- und Forschungsprogramm der NPS in seinen wesentlichen Zügen zu skizzieren. Damit soll deutlich werden, dass es jenseits der dominanten Spielart der phänomenologischen Soziologie sensu Schütz und Nachfolger eine weitere, ganz anders gelagerte phänomenologische Soziologie gibt. Dazu werden im Folgenden die zentralen Konzepte der NPS vorgestellt: Leib und Gefühl und das affektive Betroffensein als präpersonales Apriori des Sozialen (2.), leibliche Kommunikation als Grundform des Sozialen (3.) sowie die gemeinsame Situation - bestehend aus Sachverhalten, Programmen und Problemen - als sozialontologisches Fundament und empirische Erscheinungsform von Sozialität (4.). Das Fazit fasst die Kernelemente der NPS und ihr grundlegendes Erkenntnispotenzial zusammen, benennt ihre Erkenntnisgrenzen und gibt einen Ausblick auf die daraus folgende Ausarbeitung des hier vorgelegten Entwurfs (5.).

\section{Leib und Gefühl: Affektives Betroffensein als präpersonales Apriori des Sozialen}

Der basale analytische Referenzpunkt der an Husserl angelehnten, phänomenologisch fundierten Soziologie ist das Bewusstsein. Weil Wirklichkeit sich in „Bewusstseinstätigkeiten“ konstituiere (Luckmann 2008: 34), sei es Aufgabe der Soziologie, die „subjektiven Bewusstseinsgegebenheiten“ (Hitzler 2005: 233) von Menschen zu rekonstruieren, um auf diese Weise herauszuarbeiten, wie

3 Anknüpfend an Schmitz werde ich im Weiteren Neophänomenologische Soziologie mit großem N schreiben, um deutlich zu machen, dass es sich dabei um einen eigenständigen Denkansatz handelt und nicht um eine neuere Entwicklung innerhalb der traditionellen phänomenologischen Soziologie. Weil die Bezeichnung Neophänomenologische Soziologie recht lang ist, verwende ich das Kürzel NPS. je gegebene historische Wirklichkeiten durch menschliches Handeln - ebenfalls eine Bewusstseinsleistung (vgl. Schütz \& Luckmann 2003: 454) - konstruiert werden. Der Mundanphänomenologie gilt daher die „subjektive Perspektive" des menschlichen Akteurs - sein Erleben, seine Erfahrungen und sein Handeln - ,als letzter Bezugspunkt für sozialwissenschaftliche Analysen“ (Hitzler 2005: 236, Herv. im Orig.; siehe dazu auch Schütz 1971a: 50).

Die NPS teilt diese Auffassung der Mundanphänomenologie, insofern auch sie Subjektivität ins Zentrum ihrer Analysen stellt. Ihr Konzept von Subjektivität ist jedoch ein anderes. Subjektivität im neophänomenologischen Sinne meint nicht subjektives Bewusstsein, sondern „subjektive Sachverhalte (Tatsachen)“, und das sind zu allererst die Tatsachen des „affektiven Betroffenseins“ (Schmitz 1990: 6). Ein Sachverhalt ist subjektiv, „wenn höchstens einer, und zwar nur im eigenen Namen, ihn aussagen kann“ (ebd., Herv. im Orig.). Dass ich Schmerzen habe oder hungrig bin, mich vor etwas ängstige oder sehnlichst etwas begehre, weiß ich mit zweifelsfreier Gewissheit, wenn bzw. weil ich davon affektiv betroffen bin. Andere, die mich in meinem Schmerz oder Hunger erleben oder mich so gut kennen, dass sie wissen, wovor ich mich ängstige oder was ich begehre, können darüber nur im Sinne „objektiver“ bzw. „neutraler“ Tatsachen reden (ebd.: 5). Nichts von alldem wird für sie die subjektive Bedeutung haben, die es für mich hat, weil diese Phänomene nur mir wirklich nahe gehen, weil nur ich von ihnen tatsächlich betroffen bin. Subjektive Tatsachen sind Sachverhalte, die keinen Zweifel daran lassen, dass es um einen selbst geht - im Sinne des Ausspruchs von Horaz: „tua res agitur“ (ebd.: 6). Die Tatsachen des affektiven Betroffenseins besitzen damit maximale Subjektivitätsevidenz. Schmitz schlussfolgert daraus, dass das affektive Betroffensein „das Wichtigste im Leben“ der Menschen sei, eben weil sich an ihm zeige, ,was Menschen wichtig nehmen, wofür und wogegen sie sich mit Wärme einsetzen“ (Schmitz 2003: iii). Da es seiner Philosophie ganz wesentlich um die Rehabilitierung des Subjektiven geht, steht das affektive Betroffensein „im Zentrum der Neuen Phänomenologie“ (ebd.).

Die NPS folgt dieser Auffassung und begründet damit ihr normatives Ziel: Weil für Menschen Phänomene des leiblich-affektiven Betroffenseins das Wichtigste im Leben sind, hat sich die Humanwissenschaft Soziologie diesen Phänomenen zu widmen. Darüber hinaus hat die Entscheidung für das Primat des leiblich-affektiven Betroffenseins den soziologischen Vorteil, dass damit der Kreis sozial relevanter Akteure sehr weit gefasst werden kann, weiter jedenfalls, als dies in der Soziologie üblich ist. Denn affektives Betroffensein gibt es zum einen bereits 
auf präpersonaler Ebene, nämlich bei Menschen, die in Ermangelung der Fähigkeit zur Selbstzuschreibung noch nicht oder nicht mehr Personen ${ }^{4}$ sind, beispielsweise Säuglinge und Demente, womöglich auch Komatöse, zum anderen auf prähumaner Ebene, also bei Tieren, zumindest bei höher entwickelten Tieren. Sie alle sind im Sinne der NPS, die das leiblich-affektive Betroffensein als Apriori des Sozialen betrachtet, (potenzielle) soziale Akteure.

Von einem Apriori des Sozialen ist hierbei in dem Sinne die Rede, dass das leiblich-affektive Betroffensein die Bedingung der Möglichkeit von Sozialität ist. Wo niemand affizierbar ist, also keine Leiber, sondern nur leblose Körper involviert sind, ist Sozialität nicht möglich. Sozialität setzt Leiblichkeit voraus. Leiblichkeit ist das Apriori des Sozialen konkret deshalb, weil das affektive Betroffensein auf etwas oder jemand Anderen verweist: Ein leibliches Ego ist von Alter affiziert (betroffen, ergriffen), wobei Alter nicht notwendigerweise ein leibliches Wesen und damit auch kein anderer Mensch sein muss (s. dazu Abschnitt 3.3). Leiblichkeit in dem pathischen Sinne des affektiven Betroffenseins ist eine relationale Kategorie, in der das Eigene (Leib) mit dem Anderen (fremde, auch nichtmenschliche Körper, ebenso Immaterielles) verbunden ist. Wie in Abschnitt 3 gesagt werden wird, ist die darauf aufbauende leibliche Kommunikation daher die ursprüngliche Einheit des Sozialen.

Mit der Annahme, Leiblichkeit sei das Apriori des Sozialen, steht die NPS keineswegs allein. Schon früh hatte Lindemann in ihren geschlechtersoziologischen Studien gezeigt, dass passive Leiberfahrungen für die Stabilisierung der Geschlechterordnung wesentlich sind (vgl. Lindemann 1992, 1993), was sie in ihren jüngsten sozialtheoretischen Arbeiten dahingehend verallgemeinert hat, dass jede soziale Ordnung in raumzeitlich verfassten leiblichen Umweltbeziehungen wurzelt, von denen das Selbst spürbar betroffen ist (vgl. Lindemann 2014). Bei Hartmut Rosa wiederum ist die Rede davon, dass die „Präsenz von Welt [..] grundsätzlich eine pathische“ (Rosa 2016: 188; Herv. im Orig.) sei, während Waldenfels gar von der „Geburt des Sozialen aus dem Pathos“ (Waldenfels 2015: 76) spricht (zu Rosa und Waldenfels vgl. Gugutzer 2017b).

Der Fokus der NPS auf das leiblich-affektive Betroffensein als Apriori des Sozialen entspricht also keinem Alleinstellungsmerkmal. Die NPS kann jedoch für sich

4 Eine Person ist nach Schmitz ein „Bewußthaber [= Subjekt; R.G.] mit Fähigkeit zur Selbstzuschreibung. Selbstzuschreibung besteht darin, sich für etwas oder [...] etwas für sich zu halten; sie ist das spezifisch personale Selbstbewusstsein (oder Sichbewußthaben)“ (Schmitz 2003: 64; Herv. im Orig.). Zu Schmitz’ Personentheorie siehe insbesondere Schmitz (1980a). reklamieren, diesen Aspekt zu einer zentralen handlungstheoretischen Kategorie ausformulieren zu können. Mit Jürgen Hasse lässt sich diese Kategorie als „Patheur“ bezeichnen. Hasse versteht darunter „das vitalen und intuitiven Impulsen folgende Individuum im Unterschied zum rationalen und planvoll handelnden ,Akteur.“ (Hasse 2011: 70) $)^{5}$ Handlungstheoretisch übersetzt heißt das, dass die Kategorie Patheur ein antirationalistisches, antiteleologisches Handlungsverständnis repräsentiert. Es weist darauf hin, dass soziales Handeln in vielen Fällen ein leiblich angeleitetes und körperlich ausgeführtes Handeln jenseits willentlicher Kontrolle ist. In diesem Sinne lässt es sich auch als (eigensinnig) leibliches Handeln bezeichnen (vgl. Gugutzer 2012: 53 ff.; s. dazu auch Abschnitt 4.2).

Ein sozialer Patheur zeichnet sich des Weiteren dadurch aus, dass sein Handeln maßgeblich von dem ihn umgebenden Raum und der ihn dadurch affizierenden Atmosphäre motiviert ist. „Ein Patheur benutzt einen Raum nicht wie ein Autofahrer die Straße auf dem Weg zu einem angestrebten Ziel. Er ermächtigt vielmehr eine ,herumwirklich' ausgedehnte Atmosphäre, über seine persönliche Situation an dieser Stelle im Raum Macht zu entfalten und Einfluss auf sein Tun zu gewinnen“ (Hasse 2010: 70). Der soziale Patheur ist eingebunden in eine räumlich ausgedehnte, atmosphärisch aufgeladene Situation, von der er auf eine Weise berührt wird, dass sie Auswirkungen auf sein Handeln und infolgedessen auf die Situation hat. Für die soziologische Handlungstheorie folgt daraus, dass mit dem Fokus auf den sozialen Patheur ,automatisch' auch die - handlungstheoretisch quasi inexistenten - Kategorien Raum und Atmosphäre in den Blick kommen.

Schließlich bzw. zu allererst lässt sich mit der Kategorie des sozialen Patheurs der vorherrschende aktivistische Handlungsbegriff der soziologischen Handlungstheorie relativieren. Soziales Handeln ist nicht nur ein bewusstintentionales Agieren, sondern ein gleichermaßen passiverlittenes Tun. Die phänomenologisch fundierte Handlungstheorie trägt dem zwar einerseits Rechnung, indem sie „Zwischen dem Erleiden von Auferlegtem und dem Bewirken von Verfügbarem“ unterscheidet (Luckmann 1992: 28). Doch unterlässt sie es andererseits aufgrund ihrer Bezugnahme auf Husserls Bewusstseinsphilosophie, die Handlungsdimension „Erleiden“ leibphänomenologisch zu konkretisieren und dem passiv-pathischen leiblichen

5 Im Konzert soziologischer Akteursmodelle kommt der „emotional man“ (Flam 1990a, 1990b) dem Patheur am nächsten. Die Differenz zwischen beiden besteht darin, dass der Patheur das leiblich-affektive Moment sozialen Handelns in den Mittelpunkt rückt, während der - im Unterschied zum „pure emotional man“ empirisch bedeutsamere - „constraint emotional man“ von vornherein normativ und rational imprägniert ist (vgl. dazu Schimank 2000: 108-121). 
Handeln einen zentralen Platz in ihrer Handlungstheorie einzuräumen. Die NPS schließt diese handlungstheoretische Lücke. Indem sie ihren Blick auf die leiblichen Widerfahrnisse des Lebens richtet, fokussiert sie zudem ganz besonders das Überraschende, Unerwartete, Neuartige und somit nicht oder nur bedingt willentlich Kontrollierbare sozialer Situationen (s. Abschnitt 4.1).

Aus dem bisher Gesagten ergeben sich forschungsprogrammatische Konsequenzen für die NPS. Neophänomenologisch-soziologische Untersuchungen zeichnen sich zum einen durch empirische Gegenstandsbereiche aus, in denen das leiblich-affektive Betroffensein explizit Thema ist. Ob typische Liebesrituale in der Paarbeziehung, Streit(verweigerungs)kulturen in Unternehmen oder gesellschaftliche Folgen kollektiver Proteste von „Wutbürgern“ - soziologisch bedeutsame Phänomene des leiblichaffektiven Betroffenseins finden sich sowohl auf mikro-, meso- als auch auf makrosozialer Ebene. Zum anderen ist der theoretische Fokus der NPS immer auch auf das Handeln und Interagieren sozialer Patheure gerichtet. Selbst oder gerade bei Themen, die vermeintlich - weil zum Beispiel der soziologische Mainstream nicht darauf achtet - nichts mit Leib und Gefühl zu tun haben, richtet sie ihr Augenmerk auf die soziale Relevanz passiv-pathischer Leiberfahrungen. Akteur und Patheur sind der NPS gleich wichtig.

\section{Leibliche Kommunikation: Grundform des (transhumanen) Sozialen}

Die NPS setzt am leiblich-affektiven Betroffensein als Apriori des Sozialen an, ist damit aber noch nicht zwangsläufig auf der Ebene des Sozialen angelangt. Hunger, Durst, Angst oder Schmerz gehen dem Individuum spürbar nahe, ohne dass es dafür eines zweiten Individuums bedürfte. Der Weg von der individuellen zur transindividuellen Ebene führt über die leibliche Kommunikation (vgl. Schmitz 1978: 75-109, 2011: 29-53), die, wie oben erwähnt, auf dem relationalen Charakter des affektiven Betroffenseins basiert. Ist der Patheur das zentrale Subjekt der NPS, so die leibliche Kommunikation ihr primordiales Sozialitätskonzept.

Grundlage des Konzepts leibliche Kommunikation ist die räumlich-dynamische Struktur des Leibes (vgl. Schmitz 2011: 7-27). Als dessen basales Gegensatzpaar bezeichnet Schmitz „Enge“ und „Weite“: Das leibliche Befinden schwankt stets „zwischen dem Maximum möglicher
Enge (bloßer primitiver Gegenwart) und dem Maximum möglicher Weite (reiner, maßloser Weite) in tausendfältigen Abwandlungen, ohne je ganz einen dieser Pole zu erreichen." (Schmitz 1965: 75) Die leibliche Dynamik von „Engung“ und „Weitung“ bildet den vitalen Antrieb des Menschen. Im Wachzustand befindet sich der Mensch leiblich stets zwischen diesen beiden Polen, wobei etwa im Erschrecken, in der Angst oder bei Hunger die Engung überwiegt, beim Einschlafen, entspannten Dösen oder nach dem Orgasmus die Weitung. Solange der Mensch bei Bewusstsein ist, stehen Engung und Weitung in einem dynamischen Konkurrenzverhältnis, was heißt, dass sie gegeneinander ,arbeiten', ohne voneinander los zu kommen. Konkurrieren Engung (bzw. „Spannung“) und Weitung (bzw. „Schwellung“) „simultan“ um die Vorherrschaft, ist dies als leibliche „Intensität“ spürbar, zum Beispiel in der Kraftanstrengung; ist ihr Widerstreit „sukzessiv“, resultiert daraus ein leiblicher „Rhythmus“, beispielsweise in der Wollust. Vermittelt wird der Dialog von Enge und Weite durch die „leibliche Richtung“, die unumkehrbar von der Enge in die Weite führt, exemplarisch im Blick oder beim Ausatmen (vgl. ebd.: $75-126){ }^{6}$

Soziologisch relevant wird dieser innerleibliche Dialog zwischen Engung und Weitung nun dadurch, dass er in einen transsubjektiven leiblichen Dialog übergehen kann. Das ist der Fall, wenn das leibliche Subjekt von Etwas oder Jemanden außerhalb seiner selbst in einem Maße affiziert wird, dass es sich dazu verhalten muss. Leibliche Kommunikation in diesem allgemeinen Sinne liegt also vor, „wenn jemand von etwas in einer für ihn leiblich spürbaren Weise so betroffen und heimgesucht wird, daß er mehr oder weniger in dessen Bann gerät und mindestens in Versuchung ist, sich unwillkürlich danach zu richten und sich davon für sein Befinden und Verhalten in Erleiden und Reaktion Maß geben zu lassen." (Schmitz 1978: $31 \mathrm{f}$.)

Das Zitat deutet an, dass es leibliche Kommunikation bzw. „Einleibung“, der Haupttyp leiblicher Kommunikation, keineswegs nur zwischen Lebewesen gibt, denn in den Bann geraten kann ein Mensch auch von einem Spielfilm oder dem Blick aufs Meer. In Fällen wie diesen handelt es sich um „einseitige Einleibung“; sie ist charakteristisch für alle Situationen der Faszination oder Suggestion durch

6 Bei den genannten Begriffen handelt es sich um die Strukturmerkmale des Leibes, die Schmitz auch als „Alphabet der Leiblichkeit“ (Schmitz 1965: 169 ff.) bezeichnet. Neben den hier genannten ,Buchstaben“ finden sich darin außerdem die „Leibesinseln“ (spürbare Regionen am Körper), von denen die meisten temporär sind (z.B. Jucken an der Schulter, Kopfweh), sowie die „protopathische Tendenz“ (zum Dumpfen, Diffusen, Strahlenden) und die „epikritische Tendenz“ (zum Spitzen, Scharfen) (vgl. ebd.: 143-169). 
Nichtmenschliches (Musik, Bilder, Räume, Landschaften etc.). Da es sich dabei um ein im wörtlichen Sinne Bezogensein des faszinierten Menschen auf Etwas außerhalb seiner selbst handelt, lässt sich bereits auf der Ebene einseitiger Einleibung von einer sozialen Beziehung sprechen. Dies jedenfalls dann, wenn man die etymologische Bedeutung des Wortes „sozial“ ernst nimmt, der zufolge „socius“ „gemeinsam“, „gemeinschaftlich“, „verbunden“ (bzw. „Verbündeter“, „Gefährte“, „Kamerad“) meint. Verbunden ist der Mensch auch mit der Musik, die ihm in die Glieder fährt und zum tanzenden Sich-Bewegen verführt.

„Wechselseitige Einleibung“ wiederum liegt vor, wenn sich die beteiligten Interaktionspartner gegeneinander affizieren und dies Auswirkungen auf ihr Agieren hat. Ist die Art der leiblichen Kommunikation eher ein Gegeneinander, spricht Schmitz von „antagonistischer“ wechselseitiger Einleibung; ist sie eher ein Miteinander, handelt es sich um „solidarische“ wechselseitige Einleibung. ${ }^{7}$ Ein alltägliches und sozial ungemein bedeutsames Beispiel für antagonistische Einleibung ist der Blickkontakt, den Schmitz (1990: 136) auch als einen leiblich ergreifenden „Ringkampf“ beschreibt. „Der Austausch von Blicken ist ein auf leiblichem Terrain ausgetragener Machtkampf, einerlei, ob ihn die Beteiligten führen wollen oder nicht." (Gugutzer \& Holterman 2017: 271) Im Unterschied dazu ist die leibliche Kommunikation beim Singen im Chor oder beim Rudern im „Achter“ deutlich solidarischer: Es gibt hier einen „gemeinsamen vitalen Antrieb“, der die Interaktionspartner miteinander verbindet, ohne dass diese sich explizit einander zuwenden müssten; entscheidend ist, dass sie mit dem Singen bzw. Rudern ein gemeinsames, „integrierendes Thema“ haben (Schmitz 2011: 47).

Die genannten Formen der Einleibung (ein-/wechselseitig, antagonistisch/solidarisch) haben gemein, dass sie sich in vermittelter Weise ereignen. Einleibung wird ermöglicht durch Medien leiblicher Kommunikation, konkret durch „leibnahe Brückenqualitäten, die sowohl am eigenen Leib gespürt als auch an begegnenden Gestalten wahrgenommen werden können." (ebd.: 33) Als wichtigste Medien bzw. „Brücken leiblicher Kommunikation“ (Schmitz 2005: 168-184) bezeichnet Schmitz zum einen „Bewegungssuggestionen“, etwa den Rhythmus, zum anderen „synästhetische Charaktere“, also die „sogenannten intermodalen, die verschiedenen Sinnesgebiete (Se-

7 Anstelle von „antagonistischer“ und „solidarischer Einleibung“ spricht Schmitz gelegentlich von „,asymmetrischer“ und „symmetrischer Beziehung “ (Schmitz 1980c: 39 f.). Eberlein (2013: 97) übersetzt diese Ausdrücke mit „entgegengesetzter“ und „gleichgerichteter Dynamik“, Landweer (2016: 155 f.) mit „bipolarer“ und „unipolarer leiblicher Interaktion“. hen, Hören, Tasten etc.) quer verbindenden Qualitäten“ (ebd.: 176). Warm oder kalt kann die Lufttemperatur wie auch eine Stimme sein, weich oder hart ein Kissen oder ein Blick, dunkel oder hell die Nacht oder ein Pfiff, und all das kann das individuelle und soziale Handeln mitunter massiv beeinflussen.

\subsection{Wechselseitige Einleibung als Quelle der Du-Evidenz}

Das Konzept der leiblichen Kommunikation ist für die Soziologie zum einen bedeutsam, weil es eine empirische Grundlage für soziale Beziehungen zwischen Menschen und nicht-menschlichen Entitäten liefert. Zum anderen hat es eine grundlagentheoretische Relevanz in der Hinsicht, dass es das für die phänomenologische Soziologie zentrale Intersubjektivitätsproblem zu lösen hilft. Gemeint ist damit die Frage, wie der Zugang zum Anderen möglich ist, wie Ego und Alter ein soziales Verhältnis eingehen und sich einander erkennen können. Schütz hat diese Frage bekanntlich unter dem Gesichtspunkt des „Fremdverstehens" behandelt und sie mit der strukturellen Ähnlichkeit der Bewusstseinsprozesse bei Ego und Alter sowie mit der „Generalthese der Reziprozität der Perspektiven“ beantwortet (vgl. Schütz 1960: §§ 19-28). Dem zufolge versteht Ego den Sinn der Handlung von Alter näherungsweise, weil bzw. insofern er die Zeichen und Anzeichen, die Alter als Ausdrücke seines Bewusstseins vermittelt, interpretiert. Für das alltägliche Handeln der Menschen reicht dieser pragmatische Zugang zum Anderen, dessen Kern die Typisierung von Handlungsmotiven ist. Schütz hat das von Husserl transzendentalphänomenologisch bearbeitete Intersubjektivitätsproblem nach seiner Abkehr von Husserl (vgl. Srubar 2007: 173-193) somit mundanphänomenologisch gelöst: Intersubjektivität lässt sich nicht vom transzendentalen Subjekt aus erschließen, weil es keinen unmittelbaren Weg vom einen Bewusstsein zum anderen gibt, sie lässt sich aber lebensweltlich erschließen, weshalb Schütz von einer „mundanen Intersubjektivität“ (Schütz 1971c: 138) spricht.

Aber ist damit die Frage der Konstitution von Intersubjektivität wirklich beantwortet? Wie Nico Lüdtke (2008) zu Recht anmerkt, eher nicht. Mit seiner „Generalthesis des alter ego" setzt Schütz die lebensweltliche Existenz anderer Menschen nämlich als gegeben voraus, womit sich die „Frage, wie sich ego und alter wechselseitig als Sozialwesen erfassen und auf dieser Grundlage ein dauerhaftes intersubjektives Band erzeugen können“, gar nicht mehr stellt (ebd.: 192). Mit dem Konzept der mundanen Intersubjektivität wird also zum einen nicht ersichtlich, was 
die Voraussetzungen der Sozialität von Ego und Alter sind, zum anderen impliziert es, dass Sozialwesen ausschließlich Menschen sind. Lüdtke schlussfolgert daraus etwas resigniert, dass das „Problem der Intersubjektivität [..] in der Phänomenologie nach wie vor ungelöst“ (ebd.: 193) sei bzw. „seiner Lösung harrt“ (ebd.: 195).

Diese Einschätzung trifft allerdings nicht auf die Neue Phänomenologie zu. Aus neophänomenologischer Perspektive krankt der Husserl'sche wie auch der Schütz'sche Versuch, das Intersubjektivitätsproblem zu lösen, daran, dass beide den pathischen, spürbar-spürenden Leib nicht kennen, ${ }^{8}$ sondern nur den (Leib-)Körper einerseits, Geist bzw. Seele oder Bewusstsein andererseits. Im Schmitz'schen Verständnis stehen sie damit in der vorherrschenden Tradition der „psychologistisch-reduktionistisch-introjektionistischen Denkweise“ der europäischen Intellektualkultur (Schmitz 2003: 133), die die Frage offen lässt, „wie Menschen zueinander und zu den Dingen kommen.“ (ebd.) Husserl wie Schütz gebrauchen zwar das Wort Leib, meinen damit aber den lebendigen Körper oder den fungierenden (aktiven) Leib, nicht jedoch den Leib im Sinne des affektiven Betroffenseins, des Sich-Spürens. Ego und Alter werden von ihnen als zwei fest umgrenzte, abgeschlossene Körper mit einer von außen unzugänglichen Innenwelt, dem Sitz des Bewusstseins, konzipiert, und der einzige Weg, die Kluft zwischen sich und dem Anderen zu überbrücken, besteht für Ego und Alter in der Deutung der wahrnehmbaren Zeichen des Anderen (bzgl. Schütz siehe Coenen 1985b: 201 ff., bzgl. Husserl vgl. Schmitz 2003: $146 \mathrm{f}$.).

Aus Sicht der Neuen Phänomenologie löst sich das Intersubjektivitätsproblem hingegen im Medium leiblicher Kommunikation: Die Brücke zwischen Ego und Alter schlägt die wechselseitige Einleibung. Oder wie Schmitz sagt: „Wechselseitige Einleibung [...] ist die Quelle der Du-Evidenz, mit einem Bewußthaber als Partner zu tun $\mathrm{zu}$ haben, sei er nun ein anderer Mensch oder ein Tier." (Schmitz 2003: 39 f.; Herv.: R.G.; siehe auch ebd.: 151 sowie 2011: 42f.) Grundlage hierfür ist das affektive Betroffensein von Ego durch Alter (und vice versa). Darauf hatte bereits Sartre in seiner Analyse des „Blicks“ hingewiesen, in der er darlegte, dass das Getroffensein vom Blick des Anderen, das als subjektives Betroffensein von diesem erfahren wird, als Nachweis der Existenz des Anderen genü-

8 Zwar hat Husserl in den „Ideen II“ die Subjektivität des Leibes in Abgrenzung zum Körper als Objekt herausgearbeitet, paradigmatisch illustriert am Beispiel der „Tastempfindungen“ (Husserl 1952: 145 ff.), doch bleibt sein Leibverständnis letztlich ein aktivistisches, der Leib als fungierender Leib, nicht jedoch ein pathisches (vgl. Böhme 2003: 20 f.). Das leibliche Spüren ist Husserls wie auch Schütz' Thema nicht. ge (vgl. Sartre 1993: 299-397). Schmitz bezeichnet Sartres „Betroffenheitstheorie“ daher auch als erste Theorie, die das affektive Betroffensein als Basis „ursprünglicher Partnerfindung“ herausgearbeitet habe (Schmitz 1980b: 88). Allerdings habe Sartre die Wechselseitigkeit des Blicks übersehen und so getan, als reiche zum Beispiel die durch das Angeblicktwerden des Anderen empfundene Scham, um bereits ,beim' anderen Subjekt zu sein. Für die ursprüngliche Partnerfindung - soziologisch formuliert: die Konstitution von Intersubjektivität - ist jedoch wechselseitige Einleibung konstitutiv: Man fühlt sich berührt vom Anderen, spürt etwa dessen Blick am eigenen Leib, wie man auch am Anderen leibliche Regungen oder sonstige Ausdrücke unmittelbar spürbar - also ohne bewusste Zeicheninterpretation - wahrnimmt, weil diese über Bewegungssuggestionen (z. B. Geste) oder synästhetische Charaktere (bspw. sanfte Stimme) direkt vermittelt sind (vgl. ebd.: 90 f.).

Der Blick ist selbstredend nicht die einzige Form leiblicher Kommunikation, in der sich Intersubjektivität konstituiert, gleichwohl ist er ein ausgezeichnetes Beispiel dafür, wie ein wechselseitiges Aufeinanderbezogensein ohne Projektionen, Apperzeptionen, Analogieschüsse oder ein Einfühlen in den Anderen auskommt. Im Blickkontakt haben Ego und Alter die „unwillkürliche Gewissheit, mit einem anderen Bewussthaber zu tun zu haben.“ (Schmitz 2011: 41) Georg Simmel spitzt diese Annahme soziologisch zu, wenn er davon spricht, dass das Auge bzw. der Blick „die unmittelbarste und reinste Wechselbeziehung [ist], die überhaupt besteht.“ (Simmel 1992: 723) Der Blick kann daher auch als „ursprüngliche Quelle von Sozialität“ (Gugutzer \& Holterman 2017: 268) verstanden werden, wechselseitige Einleibung entsprechend als Grundform des Sozialen.

Zusammenfassend heißt das: Das Intersubjektivitätsproblem klärt sich aus Sicht der Neuen Phänomenologie in einer Erweiterung und ,Tieferlegung ' des Verhältnisses von Ego und Alter. Während die egologische Perspektive vom Bewusstsein Egos auf jenes von Alter gerichtet ist (E $\rightarrow$ A) und damit scheitert, richtet sich die neophänomenologische Perspektive auf Egos von Alter in Anspruch genommene Leiblichkeit $(\mathrm{E} \leftarrow \mathrm{A})$ und gleichzeitig auf Alters von Ego in Anspruch genommene Leiblichkeit $(\mathrm{E} \rightarrow \mathrm{A})$. Weil das leiblich-affektive Betroffensein das Apriori des Sozialen ist, ist die darauf aufbauende leibliche Kommunikation vom Typ der wechselseitigen Einleibung das hinreichende Kriterium für die Evidenz, dass es Andere für mich und mich für Andere gibt $(\mathrm{E} \leftrightarrow \mathrm{A})$. Die Lösung des Intersubjektivitätsproblems lautet für die NPS also nicht mundane Intersubjektivität, sondern leibliche Intersubjektivität. 


\subsection{Transhumane Soziologie}

In den beiden vorangegangenen Abschnitten klang wiederholt an, dass leibliche Kommunikation keineswegs nur zwischen Menschen stattfindet, sondern ebenso sehr zwischen Menschen und Tieren wie auch zwischen Menschen und leiblosen Gegenständen. Der treuherzige Blick seines Hundes rührt den Menschen so sehr, dass er sich von seinem Vorhaben, diesem gegenüber ,hart' zu bleiben, abrückt und ihm sofort verzeiht (vgl. Gugutzer \& Holterman 2017); das neue Auto muss von seiner Besitzerin erst ,eingefahren' werden, bevor sie sich darin wohlfühlt und weiß, wie es ,so tickt'; für den Fußballer ist die Schwere und für den Basketballer die Glätte des Balles essenziell, um gute Pässe spielen zu können (vgl. Gugutzer 2015b). Auch „Halbdinge“9 (Schmitz 1978: 116-132, 1990: $216 \mathrm{ff}$.) wie der Wind beim Tennis oder die angespannte Atmosphäre im Klassenzimmer sind für soziale Prozesse von Bedeutung, weil bzw. sofern sie die Leiber der Beteiligten ergreifen. Die Beispiele des Autos, der Bälle und der Atmosphäre weisen darauf hin, dass wechselseitige Einleibung auch „im Umgang mit unbelebten Gegenständen“ vorkommt (Schmitz 1990: 139). Wechselseitig ist die Einleibung in diesen Fällen, weil auch Auto, Ball und Atmosphäre auf den Patheur reagieren: Je nach dessen Verhalten stirbt das Auto ab, verspringt der Ball und kippt die Atmosphäre, oder auch nicht.

Hieran lässt sich ablesen, dass die NPS ein sehr weites Verständnis vom Sozialen hat. In den Grenzen des Sozialen befinden sich für die NPS keineswegs nur Menschen, sondern ebenso Tiere, Dinge und Halbdinge. In diesem Sinne versteht sie sich als transhumane Soziologie (vgl. dazu besonders Uzarewicz 2011). Der Mensch ist ihr zwar der wichtigste Akteur und Patheur, nicht jedoch der einzige. Dies zum einen aus dem empirisch unstrittigen Grund, dass den Menschen auch andere Lebewesen, vor allem Haustiere, sowie nichtlebendige, gleichwohl ,geliebte Objekte" (Habermas 1999) wichtig sind.Zum anderen aus dem von Bruno Latour (2010: 123) vorgebrachten theoretischen Grund, dass es kritisch zu betrachten sei, ausschließlich solchen Entitäten den Status eines sozialen Akteurs zuzusprechen, die in der Lage sind, intentional bzw. subjektiv

9 Halbdinge sind nach Schmitz weder Dinge im vollen Wortsinn noch reine Sinnesqualitäten: „Im Gegensatz zu Sinnesdaten haben Halbdinge einen im Wechsel ihrer Gesichter beharrenden Charakter wie die Dinge; von den Dingen (,Volldingen') unterscheiden sie sich durch inkonstante Dauer und zweigliedrige Kausalität.“ (Schmitz 2003: 14) Der Wind existiert, solange er weht, wo er in der Zwischenzeit ist, ist irrelevant (inkonstante Dauer); wenn der Wind weht, ergreift er die Blätter des Baums unmittelbar, Ursache und Einwirkung fallen zusammen (zweigliedrige Kausalität). sinnhaft zu handeln. Dagegen ist, worauf Latour allerdings nicht hinweist, allein aus ethischer Sicht Einwand zu erheben, schließt ein solches Akteursverständnis doch Säuglinge und Demente unzulässigerweise aus dem Kreis sozialer Akteure aus. Mit Latour teilt die NPS hingegen die Auffassung, dass ,jedes Ding, das eine gegebene Situation verändert, indem es einen Unterschied macht, ein Akteur“ ist (ebd.; Herv. im Orig.). Da menschliche Situationen fortwährend von Dingen wie auch von Halbdingen und Tieren auf eine bedeutungsvolle Weise verändert werden, ist ihnen der Status eines gesellschaftlichen Mitglieds nicht zu verwehren.

Die forschungsprogrammatischen Konsequenzen aus dem in Abschnitt 3 Gesagten sind damit offensichtlich: Die NPS liefert grundlagentheoretische Beiträge zu Fragen der Konstitution und Konstruktion von Sozialität. Neophänomenologisch-soziologische Untersuchungen betrachten leibliche Kommunikationen als kleinste Analyseeinheit des Sozialen und richten ihr Augenmerkt insbesondere auf die nichtrationalen und nichtsprachlichen Abstimmungs- und Verständigungsprozesse in sozialen Situationen. Ihre primäre Analyseebene ist damit die mikrosoziale Ebene. Meso- oder makrosoziale Ereignisse werden entsprechend auf die Mikroebene ,herunter gebrochen'. Schließlich betreibt die NPS eine Entgrenzung des Sozialen nach ,innen' und nach ,außen', was heißt, dass ihre Studien auch soziale Akteure berücksichtigen, die noch nicht oder nicht mehr sprachkompetent und voll bewusstseinsfähig sind (Säuglinge, Demente, Komatöse), sowie Tiere, Dinge und Halbdinge.

\section{Gemeinsame Situation: Sozial- ontologisches Fundament und Erscheinungsform von Sozialität}

Bei den bisherigen Ausführungen lag der Fokus auf den leibtheoretischen Grundlagen der NPS. Die NPS ist jedoch keine ausschließlich leibbasierte Soziologie, geschweige denn dass sie eine Soziologie des Leibes (sozusagen als Sonderform der Soziologie des Körpers) wäre. Der sozialtheoretische Anspruch, den sie hat, wäre damit nicht einzuholen, blieben damit doch viele basale Themen der Soziologie wie Werte, Normen, Rollen, Macht, Strukturen, Institutionen, Organisationen und nicht zuletzt , die ${ }^{6} \mathrm{Ge}$ sellschaft unberücksichtigt. Das neophänomenologische Konzept, das diese für die Soziologie zentralen Themen einzufangen ermöglicht, liefert Schmitz' Situationstheorie (vgl. Schmitz 1977: 411-429, 1990: 65-70, 1999: 21-28, 
2005: 18-61). Soziologisch besonders bedeutsam ist dabei der aus dem ontologischen Grundbegriff „Situation“ (vgl. Schmitz 1990: 69) abgeleitete Typus „gemeinsame Situation“ (Schmitz 1980b: 23-74). Wie die Situation im Allgemeinen weist auch die gemeinsame Situation invariante Strukturmerkmale auf, aufgrund derer sie als sozialontologisches Fundament menschlicher Lebenspraxis und Lebensformen aufgefasst werden kann. Zugleich sind gemeinsame Situationen aber auch empirische Phänomene, insofern sie als je sozio-historisch-kulturelle Ausprägungen diesen universellen Merkmalen eine konkrete Form geben.

Der neophänomenologische Situationsbegriff hat für die NPS damit einen vergleichbaren Stellenwert wie der Husserlsche Lebensweltbegriff (vgl. Husserl 1954) für die phänomenologische Soziologie sensu Schütz, ist doch auch die Lebenswelt ein „ontologischer Strukturbegriff“ (Werner Bergmann in Hitzler \& Honer 1984: 57) bzw. fungiert sie als „Sozialontologischer ,Boden““ (Bühl 2002: 145). Im ontologischen Verständnis kann die Lebenswelt empirisch nicht untersucht werden, jedoch können dies die sich davon abhebenden faktischen „kleinen LebensWelten“ (Hitzler \& Honer 1984, 1988 im Anschluss an Benita Luckmann), „Teilzeit-Wirklichkeiten“ oder „Erlebniswelten“ (Hitzler 2008). Dasselbe gilt für gemeinsame Situationen. Als Analysekategorie ist der Situationsbegriff verglichen mit dem Lebensweltbegriff dabei ,feiner', weil er kleinere Analyseeinheiten in den Blick bekommt. Für die empirische Wirklichkeit ist nämlich kennzeichnend, dass die Lebenswelt „ein Geflecht ineinander verschachtelter und einander durchkreuzender Situationen“ (Schmitz 2005: 26) ist.

\subsection{Schmitz' Situationstheorie}

Menschen leben allzeit in Situationen. Situationen sind die „primären Heimstätten, Quellen und Partner“ jeglichen menschlichen Verhaltens, Erlebens, Vorstellens, Erwartens, Wünschens oder Wollens (vgl. Schmitz 2003: 91). Situationen rahmen und formen menschliches Denken, Fühlen und Tun, wie sie umgekehrt dadurch hervorgebracht werden, sich stabilisieren oder wandeln. So allgemein gesprochen, wird dies jede soziologische Situationstheorie ${ }^{10}$ unterschreiben. Schmitz' Situationstheorie

10 Einen Überblick über aktuelle, soziologische wie philosophische Situationstheorien, bietet Ziemann (2013a, 2013c). Schmitz' Situationstheorie wird in diesem Band jedoch nicht thematisiert, der Herausgeber selbst verweist lediglich indirekt darauf, indem er zwei Arbeiten von Michael Großheim $(2002,2005)$ zitiert (Ziemann aber unterscheidet sich von diesen ganz wesentlich. Denn das soziologische Situationsverständnis zeichnet sich bei allen Unterschieden durch zwei Merkmale aus, die aus der Sicht von Schmitz' Situationstheorie zu eng gefasst sind: Eine Situation im soziologischen Sinne ist raumzeitlich begrenzt und auf die Kopräsenz mindestens zweier Menschen bezogen. Pointiert kommt dies in Goffmans Situationsdefinition zum Ausdruck, der zufolge eine Situation „diejenige räumliche Umgebung [ist], und zwar in ihrem ganzen Umfang, welche jede in sie eintretende Person zum Mitglied der Versammlung macht, die gerade anwesend ist (oder dadurch konstituiert wird). Situationen entstehen, wenn gegenseitig beobachtet wird, sie vergehen, wenn die zweitletzte Person den Schauplatz verlässt.“ (Goffman 1971: 29) Damit verglichen ist Schmitz' Situationsbegriff abstrakter, was seinen Grund darin hat, dass es sich um einen ontologischen Begriff handelt.

Grundlage des Situationsbegriffs bei Schmitz ist dessen Mannigfaltigkeitslehre (erstmals: Schmitz 1964, zusammenfassend: Schmitz 2016: 74-142). Schmitz kritisiert darin die gängigen Ontologien und deren Fokus auf den Typus „numerischer Mannigfaltigkeit“. Dieser reduziere nämlich die ursprüngliche „chaotische Mannigfaltigkeit“ auf ein Netz an fest umgrenzten, zählbaren einzelnen Körpern (Singularismus) mit dazu gehörigen, innerlichen Eigenschaften (Psychologismus), die miteinander in Relationen stehen (Konstellationismus). Situationen zeichnen sich aber gerade durch eine vorgängige chaotische Mannigfaltigkeit aus, also durch eine Unentschiedenheit „hinsichtlich der Identität oder Verschiedenheit seiner Elemente mit oder von einander [sic].“ (Schmitz 1964: 312) Das Adjektiv „chaotisch“ ist hierbei natürlich nicht wertend gemeint, sondern rein formal. Situationen, und zwar auch und gerade Alltagssituationen, sind chaotischmannigfaltig insofern, als ihre sie charakterisierenden Elemente ursprünglich nicht als einzelne Elemente erscheinen, sondern als nicht voneinander abgehoben, verschwommen, diffus, undurchsichtig, widersprüchlich. Die gängige Alltags- und auch wissenschaftliche Praxis ist es, Einzelheiten aus Situationen herauszuholen und, jedenfalls in der Wissenschaft, in Zusammenhänge zu bringen. Dieses Vorgehen, „Situationen explizierend aufzuspalten und aus ihnen Konstellationen zu gewinnen“ (Schmitz 2005: 28), ist also ganz üblich und keineswegs verwerflich, darf jedoch nicht darüber hinwegtäuschen, dass nicht ,einzelne Gegebenheiten (Sinnesdaten, physi-

2013b: 10). Für eine frühere Diskussion des Situationsbegriffs, die sich hinsichtlich der behandelten Autoren kaum von jener 30 Jahre späteren unterscheidet (hinzugekommen ist eigentlich nur Hartmut Esser), siehe Hitzler \& Honer (1984: 62 ff.). 
kalische Messgrößen, Körper und dergleichen) der Grundstoff menschlicher Erfahrung [sind], sondern bedeutsame Situationen.“ (Schmitz 2016: 15) Schmitz definiert Situation dabei wie folgt:

„Eine Situation [...] ist charakterisiert durch Ganzheit (d.h. Zusammenhalt in sich und Abgehobenheit nach außen), ferner eine integrierende Bedeutsamkeit aus Sachverhalten, Programmen und Problemen und eine Binnendiffusion dieser Bedeutsamkeit in der Weise, daß die in ihr enthaltenen Bedeutungen (d.h. Sachverhalte, Programme, Probleme) nicht sämtlich im präpersonalen Erleben überhaupt nicht - einzeln sind.“ (Schmitz 2005: 22; Herv. im Orig.)

Eine Vorlesung an der Universität zum Beispiel ist eine Situation, die eine Ganzheit aufweist, insofern sie zeitlich (90 Minuten), thematisch (Lehrstoff), sozial (Dozentin/ Studenten) und räumlich (Hörsaal, Sitzordnung) in sich zusammengehalten wird und sich äußerlich von anderen sozialen Zusammenkünften abhebt. Der Sachverhalt, um den es hier geht, also das, was ist, ist die Vorlesung. $\mathrm{Zu}$ den Programmen ${ }^{11}$ dieser Situation zählen Normen wie Vorgaben für den Erwerb eines Leistungsnachweises und Wünsche etwa seitens der Studierenden, dass die Vorlesung unterhaltsam sein möge. Probleme (Überraschungen, Störungen, Krisen, Sorgen etc.) treten auf, wenn der Beamer streikt oder der Dozent aufgrund der Unruhe im Hörsaal den ,roten Faden' verliert. In der Vorlesungssituation selbst sind all diese und weitere Einzelelemente binnendiffus miteinander verwoben. Die einzelnen Elemente der Vorlesungssituation konstituieren zwar einen Sinnzusammenhang, aufgrund dessen klar ist, worum es geht, was relevant ist, aber was alles auf welche Weise wie genau zur Bedeutsamkeit der je konkreten Vorlesungssituation beiträgt, ist vorab nicht definiert. Aus der Sicht der NPS ist dabei wichtig, dass ergänzend zur Bedeutsamkeit „eine Situation alles Beliebige umfassen“ (Schmitz 1999: 21) kann, zum Beispiel auch Dinge, Atmosphären oder Tiere.

Wie Michael Großheim (2005: 141) treffend bemerkt, besteht ein „Vorteil der Abstraktheit“ von Schmitz' Situationsbegriff darin, dass „man auf diese Weise der vorhandenen Vielfalt von Situationen gerecht werden kann.“

11 „Programme“ umfassen Normen und Wünsche. Eine Norm ist ein Programm, das darauf hinweist, was (nicht) sein soll, was heißt, dass sie ein „Programm für möglichen Gehorsam“ (Schmitz 2012: 11) ist; Regeln, Ge- und Verbote, Ideologien etc. sind Beispiele für Normen. Ein Wunsch verweist demgegenüber darauf, ,dass etwas sein möge“ (Schmitz 2016: 132), wozu Motive, Hoffnungen, Ideale oder Sehnsüchte zählen. Normen und Wünsche bilden zusammen den programmatischen Gehalt einer Situation, den Schmitz "Nomos“ nennt (Schmitz 2012: 13).
Diese Vielfalt erschließt sich, wenn man Schmitz' Klassifizierung von Situationen berücksichtigt, die auf vier bipolaren Kategorien basiert (vgl. zusammenfassend Tab. 1). „Nach der Art der augenblicklichen Gegebenheit unterscheide ich impressive und segmentierte Situationen, nach der Art des zeitlichen Verlaufs aktuelle und zuständliche.“ (Schmitz 1999: 22; Herv. im Orig.) Impressive Situationen (Eindrücke) sind solche, die ,auf einen Schlag', vorreflexiv wahrgenommen werden und in diesem Augenblick ganzheitlich erscheinen; der erste Eindruck eines Menschen oder beim Betreten einer fremden Wohnung sind dafür Beispiele. Segmentierte Situationen sind dem gegenüber solche, die „nie in einem Augenblick ganz zum Vorschein“ kommen (ebd.). In dem Sinne sind Muttersprache, Heimat, Nation, Klasse, Familie oder Generation segmentierte Situationen. Eine aktuelle Situation wiederum zeichnet aus, dass sie jetzt geschieht und ihr Verlauf in prozessuale Abschnitte unterteilt werden kann, wie das etwa bei einem Gespräch der Fall ist. Zuständliche Situationen hingegen dauern an, so dass es sinnlos wäre, sie in kleine Zeiteinheiten zu gliedern, zum Beispiel die Biografie eines Menschen.

Das dritte Gegensatzpaar ist die für die NPS wesentliche Differenz zwischen „persönlicher“ und „gemeinsamer" Situation (Schmitz 1990: 75 ff.). Die persönliche Situation (vgl. dazu ausführlich Schmitz 1980a: 287-415) wird gebildet aus dem „persönlichen Charakter“ und der „persönlichen leiblichen Disposition“ und entspricht der Persönlichkeit einer Person. Die persönliche Situation ist eingebettet in die gemeinsame Situation, die unterteilt ist in „implantierende“ und ,includierende“ Situationen (ebd.). Eine implantierende („einpflanzende“) Situation umschließt die persönliche Situation in einem Maße, dass es der Person nur mit viel Mühe, wenn überhaupt, und nur über eine längere Zeitspanne gelingt, sich aus ihr zu lösen. Die Muttersprache oder die Herkunftsfamilie sind solche implantierenden Situationen, aus der die persönliche Situation (in der primären Sozialisation) hervorwächst; die Gemeinschaft mit einem Lebenspartner ist dagegen eine implantierende Situation, in die die beteiligten Personen hineinwachsen. Verglichen damit integrieren includierende Situationen die persönliche Situation lockerer, weshalb die Freiheit größer ist, unbeschadet aus ihnen auszusteigen. In eine peer group, eine Partei oder eine Szene wächst man zwar ebenfalls hinein, indem man deren Konventionen, Ideologien oder Lebensart übernimmt, kann diese aber doch verhältnismäßig leicht wieder aufgeben. 
Tab.1: Neophänomenologische Situationstypen

\begin{tabular}{ll}
\hline Unterscheidungskategorien & Bipolare Situationstypen \\
\hline Augenblickliche Gegebenheit & impressiv - segmentiert \\
\hline Zeitlicher Verlauf & aktuell - zuständlich \\
\hline Grad der Einzelheit & persönlich - gemeinsam \\
\hline Grad der Involviertheit & implantierend - includierend \\
\hline
\end{tabular}

Für die Soziologie ist die Schmitz'sche Situationstheorie in mehrfacher Hinsicht ein Gewinn. Erstens hilft sie, die für die Soziologie klassische und wenig fruchtbare Gegenüberstellung von Individuum und Gesellschaft zu überwinden. Eine neophänomenologisch-soziologische Situationsanalyse geht von der Verwobenheit von persönlicher und gemeinsamer Situation aus. Zwar markieren die gemeinsamen Situationen den Analysefokus der NPS, dies jedoch immer in Relation zu den darin eingebetteten und/oder aus ihnen hervorgehenden persönlichen Situationen - gemeinsame Situationen bilden den „sozialen Hintergrund" (Schmitz 1980b: 46) und den sozialen Spielraum der persönlichen Situation. Mit diesem Fokus geht die Aufhebung des Gegensatzes von Handlungs- und Akteurstheorien einerseits, Struktur- und Systemtheorien andererseits einher. Die NPS praktiziert stattdessen einen methodologischen Situationismus (s. Abschnitt 4.3), der diese divergierenden theoretischen Paradigmen integriert. Damit verbunden ist zweitens eine fehlende Festlegung auf die soziale Mikro-, Meso- oder Makroebene. Positiv formuliert: Die NPS ist nicht nur offen für soziologische Themen, die sich auf einer der drei Ebenen bewegen, mehr noch integriert sie die Mikro-, Meso- und Makro-Ebene von vornherein in ihre Analysen. Dies deshalb, weil Menschen sich de facto immer zugleich in mehreren, ineinander verschachtelten Situationen befinden, und es daher der soziologischen Konstruktions- bzw. Explikationsarbeit geschuldet ist, wenn aus diesem Situationsgeflecht einzelne Aspekte herausgeholt und auf unterschiedliche Ebenen verteilt werden.

Drittens erlaubt der neophänomenologische Situationsbegriff die Konstruktion einer Typologie von Sozialitätsformen, die quer zu den gängigen soziologischen Großgruppeneinteilungen liegt und diese dadurch produktiv ergänzen. Michael Uzarewicz (2011: 259f.) hat dazu einen instruktiven Vorschlag gemacht. Er differenziert zwischen vier relativ allgemein gehaltenen Situationstypen, nämlich:

- „Gemeinschaften“ als „segmentierte, zuständliche, gemeinsame und implantierende“ Situationen, zum Beispiel Familie oder Freundschaften,
- „Soziierungen“ als „impressive, aktuelle, gemeinsame und (der Tendenz nach gemeinschaftlich) includierende“ Situationen, etwa Konzertbesucher oder Fahrgäste im Bus,

- „Sozietäten“ als „zuständliche, gemeinsame, (der Tendenz nach gesellschaftlich) includierende" Situationen, beispielsweise Schulklassen oder Betriebe, sowie die

- „Gesellschaft“ als „segmentierte, zuständliche, gemeinsame, includierende“ Situation, die „aus Bezugnahmen auf anwesende Abwesende besteht, mit denen es keine vis á vis-Kontakte und -Begegnungen zwischen persönlich Anwesenden gibt.“ (ebd.: 260)

Weitere spezielle Situationstypen wären zum Beispiel „Massen“, „Meuten“, „Trauben“, „Herden“ (ebd.: 269 ff.) oder „Panik“ und „Flucht“ (ebd.: 273 ff.). Diese Beispiele lassen erkennen, dass die neophänomenologische Situationstheorie Formen von Sozialität analytisch und begrifflich zu erfassen vermag, die der Soziologie - abgesehen von Gemeinschaft und Gesellschaft - gemeinhin entgehen. ${ }^{12}$

\subsection{Gemeinsame Situationen: Genese, Stabilisierung, Transformation}

In Abschnitt 3 wurde leibliche Kommunikation als Grundform des (transhumanen) Sozialen eingeführt. In Fortführung dessen kann nun gesagt werden, dass leibliche Kommunikation für die Genese gemeinsamer Situationen eine wesentliche Rolle spielt. Für Gemeinschaften und Soziierungen mag das unmittelbar einleuchten, doch

12 Darüber hinaus erlaubt die Schmitz'sche Situationstheorie die Reformulierung und Neuinterpretation traditioneller soziologischer Kategorien, beispielsweise der Kategorie Geschlecht. Geschlecht im neophänomenologischen Sinne ist eine gemeinsame, zuständliche, segmentierte und implantierende Situation mit spezifischen (historisch-kulturell variierenden) Programmen und typischen Problemen. Siehe dazu exemplarisch die Ausführungen in Gugutzer (2016b: $115 \mathrm{ff}$.) zu Männlichkeit als Situation, wo auch der Vorzug einer solchen Beschreibung deutlich wird. Dieser besteht zum Beispiel darin, Männlichkeit und (biologischer) Mann zu entkoppeln. Männlichkeit als Situation impliziert, dass sowohl Männer als auch Frauen (oder weitere Geschlechter) männlich ,sein` können. Wer immer sich von Männlichkeitsprogrammen angezogen fühlt und sich entsprechend dieser Programmatik empfindet oder/und verhält, ,ist‘ männlich. Mit umgekehrtem geschlechtlichen Vorzeichen und bezugnehmend auf Beauvoirs Existenzialphänomenologie spricht Iris M. Young ganz ähnlich von „Weiblichkeit als Situation“ bzw. vom „Frausein als einer Existenzweise“ und dass „nicht notwendigerweise jede Frau ,weiblich“ sein“ müsse, wie es auch „nicht unmöglich“ sei, „daß [sic] Männer in manchen Hinsichten ,weiblich“ sind.“ (Young 1993: 711) 
auch für Sozietäten und die Gesellschaft gilt, dass „Einleibung als Quelle und Nährboden gemeinsamer Situationen“ (Schmitz 1980b: 52) fungiert. Dabei bildet vor allem die antagonistische wechselseitige Einleibung das Fundament der Entstehung aktueller und daraus gegebenenfalls resultierender zuständlicher gemeinsamer Situationen. In antagonistischer wechselseitiger Einleibung entsteht ad hoc ein die daran Beteiligten übergreifender und sie vereinnahmender Kollektivleib. Hierfür braucht es keinesfalls Worte, sondern es reichen mitunter die oben erwähnten Bewegungssuggestionen (Blicke, Gesten) und synästhetischen Charaktere (das Harte, Sanfte, Wuchtige) als Medien leiblicher Kommunikation, um den Kommunikationspartnern die Gewissheit zu vermitteln, an einer gemeinsamen Situation (Warten in der Schlange) mit dazugehörigen Programmen (Norm: nicht vordrängeln, Wunsch: es möge schnell gehen) und eventuell Problemen (Vordrängler) teilzuhaben. Durch Wiederholung und damit verbundener Habitualisierung können sich aktuelle zu einer zuständlichen gemeinsamen Situation verfestigen. Das Schlangestehen ist dann eine „Institution“, ${ }^{13}$ die jedes Mal aufs Neue „inszeniert“ (Schmitz 1990: 414) wird und dadurch am Leben erhalten bleibt.

Den Prototyp dafür, wie qua antagonistischer wechselseitiger Einleibung Menschen in Kontakt miteinander treten und damit eine gemeinsame Situation herstellen, repräsentiert das Gespräch von Angesicht zu Angesicht (vgl. Schmitz 1980b: 97-109). ${ }^{14}$ In einem Gespräch kommt es nämlich nicht nur auf das Gesagte an, vielmehr spielen leibliche Faktoren wie die spürbare Frische oder Müdigkeit des Gesprächspartners, von der man mitgerissen oder eingeschläfert wird, eine ebenso wichtige Rolle für das soziale Geschehen wie die Körperhaltung, Mimik und Blicke der Beteiligten, mittels derer nicht minder um die „Definition der Situation“ (W. I. Thomas/D. S. Thomas) gerungen werden kann wie mit Worten. Der zwischenleibliche Dialog kann infolgedessen neue Sachverhalte (Themenwechsel) hervorbringen, den Nomos (programmatischer Gehalt: Verhaltenserwartung, Hoffnungen) modifizieren oder Probleme (Streit) generieren. Eine neophänomenologisch-soziologische Analyse von Gesprächssituationen berücksichtigte dabei außerdem die persönlichen Situationen der Akteure und deren Einbettung in segmentiert-

13 Eine Institution im Schmitz'schen Sinne ist ein handlungsleitendes „System von Regeln“, wobei diese Regeln „Zulassungsbeschränkungen, Kooperationsbeschränkungen und Rollenzuweisungen“ sein können (Schmitz 2005: 258 f.; siehe auch Schmitz 1990: 414-420).

14 Ganz ähnlich sprechen auch Peter Berger und Thomas Luckmann von der „Vis-à-vis-Situation“ als dem „Prototyp aller gesellschaftlichen Interaktion“ (Berger \& Luckmann 1969: 31). zuständliche Situationen (Herkunftsmilieu, Generationenzugehörigkeit), um Ursache, Verlauf und Ergebnis des Gesprächs möglichst umfassend - im Sinne einer ,interpretativen Situageneseforschung “ (Schmid-Degenhardt 2005) - verstehend rekonstruieren zu können.

Solidarische Formen wechselseitiger Einleibung sind empirisch seltener ${ }^{15}$ anzutreffen, gleichwohl für soziale Integration im emphatischen Sinne von großer Bedeutung. Nach Schmitz (ebd.: 109-113) ist dafür das gemeinsame Singen das Musterbeispiel. Wenn Menschen miteinander singen, resultiert daraus ad hoc ein Kollektivleib, in den die daran Mitwirkenden affektiv eingeschmolzen sind. Dies hat seinen Grund darin, dass die jeweilige binnendiffuse Bedeutsamkeit der Situation mit einer kollektiven Atmosphäre aufgeladen ist, ${ }^{16}$ die für die Beteiligten als - mal mehr, mal weniger - leibliches „Ergriffensein von Gefühlen“ (Schmitz 2014: 59) spürbar ist. Je intensiver dieses atmosphärische Ergriffensein ist, desto größer ist die Wahrscheinlichkeit, dass aus der aktuellen eine zuständliche Situation resultiert, die sich im Gedächtnis Einzelner oder Mehrerer ablagert und in der Erinnerung aufgerufen wird mit dem möglichen Effekt, Jahre später erneut gemeinschaftsstiftend zu wirken: „Weißt du noch, Berlin 99, Bruce spielt ,Badlands‘ und der Volkspark flippt aus - Wahnsinn!“

Das Beispiel weist implizit darauf hin, dass die Zugehörigkeit zu einer zuständlichen gemeinsamen Situation, hier der Fangemeinschaft, voraussetzungsvoll ist. Wer nicht weiß, wer Bruce ist oder von welchem Volkspark die Rede ist, gehört nicht zu dieser Gemeinschaft. Soziale Inklusion setzt damit „kommunikative Kompetenz“ voraus, die man durch „Anpassung in Verständnis und Verhalten an gemeinsame Situationen“ (Schmitz 1980b: 47) erwirbt. Dieses Hineinwachsen in eine gemeinsame Situation mit dem Ergebnis kommunikativer Kompetenz entspricht dem traditionellen soziologischen Verständnis von Sozialisation, mittels derer das Individuum sozial handlungsfähig wird. Einer Person, der es an kommuni-

15 Das gilt zumindest in einem engen, den gemeinsamen vitalen Antrieb in den Mittelpunkt rückenden Sinne von solidarischer Einleibung. Versteht man solidarische Einleibung jedoch in einem weiten Sinne, indem man stärker ihr Merkmal des integrierenden Themas, das das Verhalten der Betroffenen anleitet, hervorhebt, dann ist sie für alle Formen von Sozialität von Belang. Diesen Hinweis verdanke ich Gutachter/in A, der/die sagt: ,Jede Institution benötigt ein impulsgebendes übergreifendes Thema, das die Leiber gewissermaßen solidarisch ausrichtet (z. B. die Arbeitszeiten und die Wege innerhalb der Institution regelt).“

16 Zum Zusammenhang von gemeinsamen zuständlichen Situationen und den darin „aufgehängten“ (Schmitz 2014: 60) kollektiven Atmosphären siehe exemplarisch die Ausführungen von Großheim et al. (2014) zu „kollektiven Lebensgefühlen“. 
kativer Kompetenz mangelt, und zwar auch an leiblicher Kommunikationskompetenz,${ }^{17}$ muss entsprechend mit sozialer Exklusion rechnen. Das gilt nicht nur für Gemeinschaften, Soziierungen und Sozietäten, sondern ebenso für die Gesellschaft: Wer Steuern hinterzieht, wird gesellschaftlich exkludiert - er kommt ins Gefängnis.

Auch wenn, wie bisher betont, wechselseitige Einleibung die Grundlage aktueller und zuständlicher gemeinsamer Situationen ist, heißt das selbstredend nicht, dass jene die einzige Quelle zur Hervorbringung von diesen ist. Die NPS legt zwar auf die leibliche Kommunikation als Basis (und Produkt) gemeinsamer Situationen ihr besonderes Augenmerk, berücksichtigt aber gleichermaßen die humanspezifische Kompetenz zur Explikation aus Situationen. Im Modus der Explikation einzelner Bedeutungen (Sachverhalte, Programme, Probleme) aus der binnendiffusen Bedeutsamkeit von Situationen gelingt es Menschen, das Chaos der Situation zu durchdringen, zu beherrschen und die Welt in geordnete Bahnen zu lenken (vgl. Schmitz 2005: 28). Die aus der Explikation resultierenden Konstellationen machen das Leben übersichtlich(er), geregelt(er), kontrollierbar(er), indem sie soziale Ordnung herstellen. Oder wie Schmitz konkretisiert: „Im sozialen Betrieb führt dieses Konstellieren zur Ausbildung von Institutionen und Organisationen, d.h. sozialen Verhaltensmustern, die durch Regeln ein wiederholbares Verhalten von Menschen zueinander vorzeichnen." (Schmitz in: Sohst \& Schmitz 2005: 37) So ist die Ehe eine Institution, die Ordnung in die chaotische Mannigfaltigkeit partnerschaftlicher Beziehungsformen bringt, indem sie regelt, wer zugelassen ist (Ehepartner), wer für die „Spielberechtigten“ (Schmitz 1990: 414) als potenzieller „Mitspieler“ in Frage kommt (Verwandte, Freunde) und welche Rollenübernahmen dauerhaft (Vater/Mutter) oder situativ gestattet (Tanzpartnerin) bzw. verwehrt (Geliebter) sind.

Dass eine Institution wie die Ehe Bestand hat, verdankt sie zu einem womöglich nicht geringen Anteil der Einbettung in die übergeordnete Institution „Staat“ (vgl. ebd.: 425-438), insofern dieser mittels steuerlicher Erleichterungen und anderer Unterstützungsprogramme die Ehe für manche als besonders attraktive partnerschaftliche Lebensform erscheinen lässt. Eine hinreichende Begründung für die Dauerhaftigkeit der Institution Ehe ist das aber sicher nicht. Wie Bourdieu in seiner Habitus- und Feldtheorie deutlich gemacht hat, haben Institutionen nur dann Bestand, wenn sie nicht allein in Regeln objektiviert,

17 Mit Bourdieu (1985: 185) könnte man die mangelnde leibliche Kommunikationskompetenz als fehlendes „Gespür“ für die Situation bezeichnen, mit Wilhelm Schmid (1999: 199) als nicht vorhandene situative „leibliche Intelligenz“. sondern ebenso sehr im Habitus der sie „bewohnenden“ Menschen verankert sind (vgl. Bourdieu 1987: 107). In neophänomenologischer Terminologie heißt das, dass Institutionen und andere zuständliche gemeinsame Situationen für ihr Fortdauern darauf angewiesen sind, dass sie in der persönlichen Situation der darin eingebetteten Menschen Wurzeln schlagen. Das geschieht wesentlich auf dem Wege, dass die Sachverhalte, Programme und/oder Probleme dieser Situation in die ,primitive Gegenwart“18 der Person hineinwirken, sie also leiblich-affektiv betreffen, und zwar (etwa aufgrund von Wiederholungen) in einem Maße, aufgrund dessen die Situation - vor allem ihr programmatischer Gehalt - Teil der „leiblichen Disposition“19 der Person wird. Die Ehe als Institution wurzelt in diesem Sinne in den leiblich verankerten Regeln, Normen, Wertungen, Erwartungen, Befürchtungen, Hoffnungen, Sehnsüchten etc., die mit ihr verbunden sind. Entsprechend ist ,Fremdgehen' eine Bedrohung für die Institution Ehe deshalb, weil sie den Nomos dieser gemeinsamen Situation spürbar (Eifersucht, Wut, Hass) verletzt oder ganz zerstört.

Soziale Institutionen und soziale Ordnung im Allgemeinen verdanken ihre Stabilität also ganz entscheidend der Leiblichkeit - bzw. dem leiblichen Habitus (vgl. Gugutzer 2002: 119) - der sie bewohnenden Menschen. Die NPS widmet sich diesem Aspekt im besonderen Maße. Ebenso sehr gilt ihr Interesse aber auch der leiblich fundierten Transformation sozialer Ordnung. Sozialer Wandel erscheint im Lichte der NPS nicht allein und auch nicht primär als Ergebnis rational geplanten, teleologischen (kollektiven) Handelns, sondern mindestens so sehr dadurch bedingt, dass Menschen von einer gemeinsamen Situation auf negative Weise leiblich-affektiv betroffen sind. Sozialem Wandel „liegt in den meisten Fällen eine Unzufriedenheit mit der gegebenen sozialen Ordnung zugrunde, und Unzufriedenheit ist ein leibliches Befinden.“ (Gugutzer 2012: 56) Die wesentlichen Akteure sozialen Wandels sind damit Patheure: Menschen beginnen, die je gegebene, gemeinsam-zuständliche Situation zu ändern, wenn sie von dieser spürbar betroffen sind. Das gilt für die soziale Mikro-, Meso- und Makroebene gleichermaßen (vgl. ebd.: 57):

18 „Primitive Gegenwart“ (bzw. „personale Regression“) bildet zusammen mit der „entfalteten Gegenwart“ (bzw. „personale Emanzipation“) die beiden Aspekte (bzw. Dynamiken) des Personseins; vgl. dazu ausführlich Schmitz 1980a, zusammenfassend Schmitz 1990: 153-174, zum Verhältnis von Person und Leib Schmitz 2011: 71-87). 19 Schmitz verwendet den Ausdruck „leibliche Disposition“ im Sinne einer die Person prägenden, weil „ganzheitlich alle leiblichen Regungen durchdringenden und umhüllenden Atmosphäre“ (Schmitz 1980a: 292). Sie ist gewissermaßen die leiblich-affektive Anlage einer Person, die ihrem Tun eine typische ,Färbung‘ verleiht. 
Paare entschließen sich, an ihrer Beziehung zu arbeiten, wenn die eine Person unter der fortdauernden Gleichgültigkeit der anderen so sehr leidet, dass das Beziehungsende droht; Firmen initiieren ein Coaching- und Mediationswochenende für ihre Mitarbeiter, wenn das wechselseitige Mobbing in der Abteilung solche Ausmaße angenommen hat, dass keine produktive Zusammenarbeit mehr möglich ist; tausende von Menschen gehen auf die Straße und demonstrieren, weil die sozialen und politischen Missstände in ihrem Land unerträglich für sie geworden sind. Sofern es in solchen Fällen zur Transformation der Paar-, Abteilungs- oder politischen Ordnung kommt, hat das wesentlich damit $\mathrm{zu}$ tun, dass einzelne oder mehrere Patheure ,das Heft in die Hand genommen` haben.

\subsection{Methodologischer Situationismus}

Die methodologische Konsequenz, die sich aus der situationstheoretischen Fundierung der NPS ergibt, lässt sich als methodologischer Situationismus bezeichnen: Gemeinsame Situationen sind die zentrale Analyseebene der NPS (während leibliche Kommunikation ihre kleinste Analyseeinheit ist). Die NPS untersucht gemeinsame Situationen auf mikro-, meso- und makrosozialer Ebene, wobei der empirischen Sozialforschung allein Gemeinschaften, Soziierungen und Sozietäten zugänglich sind, nicht jedoch Gesellschaft. Gesellschaft ist empirisch nicht untersuchbar, da „anwesende Abwesende“ (Uzarewicz 2011: 260) weder beobachtet noch mündlich interviewt oder schriftlich befragt werden können. Gesellschaft als „,imaginäre‘, latente Situation“, wie Uzarewicz im Anschluss an Cornelius Castoriadis’ Buchtitel „Gesellschaft als imaginäre Institution“ (ebd.: 261) sagt, kann nur modelltheoretisch analysiert werden. Um welchen Typ gemeinsamer Situation es sich im Einzelnen dann auch immer handelt, die NPS klärt dabei den Sachverhalt, um den es geht, den programmatischen Gehalt (Nomos) der Situation und das oder die Probleme, die darin eine Rolle spielen und das gemeinsame Handeln der Akteure und Patheure motivieren, herausfordern, anregen, erzwingen etc. Es handelt sich hiermit um einen methodologischen Situationismus, der die Subjekte nicht ignoriert, sondern mit seinem Blick auf die Verschränkung von persönlicher und gemeinsamer Situation immer auch danach fragt, wie leibliche Subjekte sowohl an der Hervorbringung, Stabilisierung und Transformation von Sozialität beteiligt sind als auch von deren Nomos und Problemen geprägt bzw. geleitet werden.

Wesentlich ist zudem, dass die NPS den Fallstricken des Situationismus' bzw. Kontextualismus', der nur raumzeitlich begrenzte Sozialeinheiten und face-to-face-Inter- aktionen kennt (vgl. zur Kritik Hörning 2004: 30 f.), entgeht, da ihr Konzept der gemeinsamen Situation nicht nur aktuelle und impressive, sondern ebenso zuständliche und segmentierte Situationen umfasst. Die NPS vertritt in diesem Sinne eine - traditionell gesprochen - transsituative Situationstheorie. Zugleich ist sie eine transhumane Situationstheorie, die auch nichtmenschliche Entitäten als integrale Bestandteile von Situationen mitberücksichtigt.

Die damit verbundene forschungsprogrammatische Haltung der NPS ist verwandt mit jener von Goffman, wenn dieser sagt, dass es seiner Soziologie „nicht um Menschen und ihre Situationen, sondern eher um Situationen und ihre Menschen" gehe (Goffman 1986: 9; vgl. dazu Gugutzer 2015c: 75 f.). Wie Goffman interessiert sich auch die NPS primär für Situationen und sekundär dafür, wie diese von Menschen hervorgebracht, bewohnt, gestaltet, einverleibt etc. werden. Der thematische Ausgangspunkt empirischer Untersuchungen sind gleichwohl typischerweise Phänomene, die einzelnen Menschen nahegehen, von denen sie betroffen sind, die sie als Patheure in Anspruch nehmen. Für die Soziologie besonders relevant sind dabei problematische Sachverhalte, einerlei ob es sich um zwischenmenschliche Missverständnisse, Spannungen, Streitigkeiten etc. oder um gesellschaftliche Konflikte, Krisen, Ungerechtigkeiten etc. handelt. Ausgehend von diesen Problemen, aus denen überhaupt erst die Forschungsfrage resultiert, ${ }^{20}$ besteht die eigentliche Forschungsaufgabe dann darin, die gemeinsame Situation zu rekonstruieren, deren Problem den Untersuchungsfokus markiert. Ziel dieser Rekonstruktionsarbeit ist es, die Genese, Prozesse und Strukturen der gemeinsamen Situation (besser) zu verstehen.

Die methodische Durchführung solcher empirischen Untersuchungen erfolgt mit den Instrumenten qualitativer Sozialforschung. Dies deshalb, weil es allein mit Hilfe qualitativer Forschungsmethoden - wenigstens näherungsweise - möglich ist, die chaotische Mannigfaltigkeit gemeinsamer Situationen analytisch zu durchdringen $;{ }^{21}$

20 Die problematischen Sachverhalte müssen nicht ,dramatisch', ,gravierend' oder ,schlimm‘ sein, die entsprechenden Forschungsfragen daher auch nicht, groß‘ oder, gewichtig، (wobei natürlich zu fragen wäre, wer mit welchem Recht eine solche Bewertung vornimmt). Es kann sich auch um ,kleinere‘ Probleme bzw. Fragen handeln, etwa: „Wie gelingt die wortlose Verständigung im Paartanz?“ (Gugutzer 2008) oder „Wie entsteht Stimmung beim Public Viewing?“ (Gugutzer 2015c).

21 Unter den derzeit vorliegenden qualitativen Forschungsansätzen kommt vermutlich die „Situationsanalyse“ von Adele Clarke (2011, 2012) dem Forschungsprogramm der NPS am nächsten, insofern NPS und Situationsanalyse das generelle Erkenntnisziel teilen, „die chaotische Komplexität der dichten Verflechtungen und Permutationen 
Tab. 2: Alte versus Neue Phänomenologische Soziologie

\begin{tabular}{lll}
\hline Vergleichskriterien & Alte Phänomenologische Soziologie & Neue Phänomenologische Soziologie (NPS) \\
\hline Philosophischer Referenzautor & Edmund Husserl & Hermann Schmitz \\
\hline Art der Phänomenologie & Transzendentalphänomenologie & Neue („naive“) ${ }^{22}$ Phänomenologie \\
\hline Sozialapriori & Bewusstsein & $\begin{array}{l}\text { Leiblichkeit } \\
\text { (affektives Betroffensein) }\end{array}$ \\
\hline Zentrales Subjektkonzept & Akteur & Patheur \\
& (intentional-reflexives Handeln) & (leiblich-vorreflexives Handeln) \\
\hline Zentrales Sozialitätskonzept & Intersubjektivität & Leibliche Kommunikation \\
\hline Ontologie & Dingontologie (Konstellationismus) & Situationsontologie \\
\hline Sozialontologisches Fundament & Lebenswelt & Gemeinsame Situationen \\
\hline Methodologie & Methodologischer & Methodologischer \\
\hline Soziologie & Individualismus & Situationismus \\
\hline
\end{tabular}

die Stärke der quantitativen Sozialforschung besteht demgegenüber darin, Erkenntnisse über Konstellationen zu generieren. Die konkrete Analyse erfolgt sodann methodenplural: Mit rekonstruktiven Verfahren gilt es, den Programmgehalt der gemeinsamen Situation (Wertvorstellungen, Normen und Regeln, Erwartungshaltungen, Wünsche und Hoffnungen etc.) herauszuarbeiten sowie die darin eingebetteten persönlichen Situationen (Biografie, Persönlichkeit) der Akteure zu analysieren. Mit Hilfe deskriptiver Verfahren wiederum gilt es, die Leiblichkeit der Patheure und die Prozesse leiblicher Kommunikationen sowie die relevanten nichtmenschlichen Situationsteilnehmer zu beschreiben. Unter methodologischem Gesichtspunkt ist außerdem zu berücksichtigen, dass auch die „Forschungssituation“ einschließlich des „Forscherleibes und Forscherkörpers" selbstreflexiv zu thematisieren sind (Gugutzer 2017a; siehe dazu auch Dörpinghaus 2013: 206-263).

\section{Zusammenfassung, Grenzen, Ausblick}

Der Beitrag hatte das Ziel, das Theorie- und Forschungsprogramm der Neophänomenologischen Soziologie zu skizzieren und damit eine Alternative zur vorherrschenden, an Husserl und Schütz orientierten phänomenologi-

der jeweiligen Situation zu erfassen und zu diskutieren.“" (Clarke 2011: 210) schen Soziologie zu entwerfen. Wenngleich eine explizite Gegenüberstellung der neuen und der alten phänomenologischen Soziologie hier nicht beabsichtigt war, wurden dennoch einige grundlegende Differenzen dieser beiden Ansätze deutlich. Tabelle 2 fasst die zentralen Unterschiede zwischen der traditionellen und der Neophänomenologischen Soziologie zusammen.

Mit dieser pointierten Gegenüberstellung ist nicht beabsichtigt, die beiden phänomenologischen Positionen gegeneinander auszuspielen oder gar eine Überlegenheit der NPS gegenüber der phänomenologischen Soziologie sensu Schütz zu reklamieren. Intendiert ist allein, die Andersartigkeit der NPS zu verdeutlichen und deren Erkenntnispotenzial hervorzuheben. Letzteres lässt sich abschließend an drei Korrekturen festmachen, die die NPS an der allgemeinen Soziologie vornimmt:

Aufgrund ihres leibphänomenologischen Fundaments korrigiert die NPS erstens die „quasimentalistische Bornierung“ (Lindemann 2005: 115) der Soziologie. Sozio-

22 Schmitz hat seine Philosophie in Abgrenzung zu Husserls Transzendentalphänomenologie als (kritisch-) „naive Phänomenologie“ (Schmitz 1964: 139) bezeichnet, um deutlich zu machen, dass er Husserls Methode der transzendental-phänomenologischen und eidetischen Reduktion für unbrauchbar hält. Weil die Neue Phänomenologie ohne Wesensschau auskommt und eine dezidiert empirische Phänomenologie ist, gilt entsprechend Luckmanns Einschätzung, dass es eine phänomenologische Soziologie „nicht geben kann“ (Luckmann 1979: 196) und Phänomenologie „Proto-Soziologie“ (ebd.: 205) sei, nur für die Husserl'sche Phänomenologie. Die Ausführungen haben gezeigt, dass die Schmitz'sche Phänomenologie soziologienahe Konzepte enthält und es daher auch eine darauf aufbauende phänomenologische Soziologie gibt. 
logische Konzepte, die traditionell kognitivistisch entworfen sind, werden von der NPS um eine leibliche Dimension erweitert. Sinn, Verstehen, Wissen, Handeln, Interaktion etc. werden entsprechend als leiblicher Sinn, leibliches Verstehen, leibliches Wissen etc. konzipiert (vgl. Gugutzer 2012: 52-74). Damit einher geht zweitens die Korrektur des aktivistischen Bias' soziologischer Akteurs- und Handlungskonzepte. Die NPS fokussiert das passiv-pathische Eingebundensein der Menschen in soziale Kontexte und damit zugleich die Widerfahrnisse, Überraschungen, Irritationen, Störungen menschlichen Zusammenlebens. Vor dem Hintergrund dieser zwei Schwerpunkte erweist sich die NPS als eine „leibhaftige Soziologie“ (Bühl 2002: 200), die sich nicht mit der Vorstellung zufriedengibt, dass Gesellschaft ausschließlich in den Köpfen der Menschen existiert und bewusst-intentional hergestellt wird. Die NPS ergänzt diese rationalistisch-kognitivistische Reduktion sozialer Wirklichkeitskonstruktion um die Idee einer untrennbaren Verschränkung von Leib und Welt. Drittens korrigiert die NPS den in der Soziologie dominanten, auf menschliche Kopräsenz im Hier-Jetzt begrenzten Situationsbegriff. Der neophänomenologische Situationsbegriff geht darüber hinaus, indem er auch die Vergangenheit sowie Nichtmenschliches thematisiert, klassische soziologische Dualismen überwindet und eine empirisch angemessene Differenzierung soziologischer Gruppenkategorien ermöglicht. Angesichts dessen, dass die neophänomenologische Situationstheorie in der Soziologie mehr oder weniger unbekannt ist, stellt deren Soziologisierung vermutlich den innovativsten sozialtheoretischen Beitrag der NPS dar.

Der hier vorgelegte Entwurf einer Neophänomenologische Soziologie ist als Startpunkt einer weiteren Ausarbeitung dieses Theorie- und Forschungsprogramms zu verstehen. Die Weiterentwicklung hat sich vor allem auf jene Punkte zu konzentrieren, an denen die NPS im Moment an ihre konzeptionellen Grenzen stößt. Diese Grenzen ,verdankt' sie ihrem theoretischen Fundament, der Neuen Phänomenologie. Das gilt zum Beispiel für die Marginalisierung des Körpers. Auch wenn es sicherlich überspitzt ist, die Neue Phänomenologie „überhaupt als Leibphilosophie“ (Böhme 2003: 25) zu bezeichnen, ist der spürend-spürbare Leib für sie doch absolut zentral, während der sicht- und tastbare Körper für sie nicht wichtig ist. Die NPS tendiert dazu, diese Einseitigkeit zu übernehmen. Das aber ist sachlich unangemessen, da der Körper für die Soziologie mindestens so bedeutsam ${ }^{23}$ ist wie der

23 Das zeigt sich faktisch an dem seit zwei Jahrzehnten enorm prosperierenden Feld der Körpersoziologie (vgl. als Überblick Gugutzer et al. 2017), wie es sich auch an den nicht wenigen theoretischen Be-
Leib. Aufgabe der NPS muss es daher sein, den Körper sowie die Verschränkung von Leib und Körper in die NPS zu integrieren. ${ }^{24}$

Die Integration des Körpers in die NPS stellt eine notwendige Aufgabe auch in der Hinsicht dar, dass mit dem Körper die aktive und reflexive Seite sozialen Handelns ins Spiel kommt. In der NPS ist dieser Handlungsaspekt bislang zu wenig ausgearbeitet, da sie primär den Patheur fokussiert, kaum jedoch den intentional und rational handelnden Akteur. Da sich in Schmitz' Philosophie nur bedingt hilfreiche Begriffe und Konzepte finden, um diesen für die soziologische Handlungstheorie wichtigen Aspekt auszuarbeiten, bedarf es dazu der Anleihe aus anderen Theorien. Eine Kandidatin hierfür ist Plessners „Theorie der Positionalität“" (Plessner 1975) und der darin formulierte „Doppelaspekt“ von „Leibsein“ und „Körperhaben“. Allen voran ist es die Kategorie Körperhaben, die für die NPS erfolgversprechend ist, da mit ihr sowohl der Körper als Körperding gemeint ist, das der Mensch instrumentell und expressiv nutzen kann, als auch die humanspezifische Fähigkeit zur reflexiven Selbstdistanzierung. ${ }^{25}$

Eine offene Frage ist schließlich, inwiefern sich die NPS zu einer Gesellschaftstheorie ausarbeiten lässt. Die Stärke der NPS liegt bislang eindeutig im Bereich der Sozialtheorie. Um auch auf gesellschaftstheoretischer Ebene ein ernstzunehmender Diskussionspartner in der Soziologie zu werden, müsste sie ihre Situationstheorie weiter ausbauen. Gesellschaft lediglich als einen spezifischen Situationstypus zu beschreiben (s. 4.1), ist zu wenig. Ein vielversprechender Ansatzpunkt wäre es, Schmitz' Situationstheorie mit seiner Normentheorie, die eine Institutionen-, Organisations- und Staatstheorie miteinschließt (vgl. Schmitz 2012), zu verbinden. Hinsichtlich einer historisch angelegten, kritischen Gesellschaftstheorie könnte außerdem Schmitz' Kritik an der zunehmenden gesellschaftlichen Tendenz zum „Konstellationismus“, die er auch als „autistische Verfehlung des abendländischen Geistes“ (Schmitz 1999: 55 ff.) bezeichnet, aufgegriffen und ausgearbeitet werden. Schmitz meint damit die „Tendenz zur Isolierung und Nivellierung der Individuen durch Auflösung implantierender Situationen zu Gunsten ver-

mühungen zeigt, den Körper als soziologischen Grundbegriff oder Verkörperung als basales Konzept der Soziologie zu etablieren (vgl. zusammenfassend Gugutzer 2012, 2015a).

24 Vorschläge zur Integration des Körpers in die NPS finden sich in Gugutzer (2012, 2015a: 20 ff.). Zur Verschränkung von Leib und Körper siehe außerdem Böhme (2003: 294 ff.) und Lindemann (1996, 2017).

25 Hierfür kann auf vorliegende Arbeiten zur Verknüpfung von Schmitz und Plessner zurückgegriffen werden: vgl. Gugutzer (2015a), Jäger (2004), Lindemann (1993). 
meintlicher Selbstbestimmung des Einzelnen“ (Schmitz 2005: 29), was sich mit traditionellen soziologischen Konzepten als Enttraditionalisierung, Säkularisierung, Individualisierung oder Atomisierung umschreiben lässt.

Neben diesen theorieimmanenten Verfeinerungen wird es für die Wahrnehmung und Anerkennung der NPS auch über den Kreis phänomenologisch interessierter Soziologinnen und Soziologen hinaus vermutlich am Wichtigsten sein, empirische Studien im Sinne des methodologischen Situationismus ' vorzulegen. Deren Ziel muss eine Hermeneutik gemeinsamer Situationen sein, die zu einem besseren Verstehen der Komplexität sozialer und gesellschaftlicher Wirklichkeit beiträgt. Dieser Artikel stellt das dafür notwendige, theoretisch-begriffliche sowie methodologisch-methodische Grundgerüst bereit.

\section{Literatur}

Abraham, A., 2002: Der Körper im biographischen Kontext. Ein wissenssoziologischer Beitrag. Opladen: Westdeutscher Verlag.

Alkemeyer, T., 2015: Verkörperte Soziologie - Soziologie der Verkörperung. Ordnungsbildung als Körper-Praxis. Soziologische Revue 38: 470-502.

Berger, P. L. \& T. Luckmann, 1969: Die gesellschaftliche Konstruktion der Wirklichkeit. Eine Theorie der Wissenssoziologie. Frankfurt am Main: S. Fischer.

Bird, G., 2009: What is Phenomenological Sociology Again? Human Studies 32: 419-439.

Böhle, F. \& D. Fross, 2009: Erfahrungsgeleitete und leibliche Kommunikation und Kooperation in der Arbeitswelt. S. 107-126 in: T. Alkemeyer, K. Brümmer, R. Kodalle \& T. Pille (Hrsg.), Ordnung in Bewegung. Choreographien des Sozialen. Körper in Sport, Tanz, Arbeit und Bildung. Bielefeld: transcript.

Böhle, F. \& S. Porschen, 2011: Körperwissen und leibliche Erkenntnis. S. 53-67 in: R. Keller \& M. Meuser (Hrsg.), Körperwissen. Wiesbaden: VS.

Böhme, G., 2003: Leibsein als Aufgabe. Leibphilosophie in pragmatischer Hinsicht. Kusterdingen: Die Graue Edition.

Bongaerts, G., 2003: Eingefleischte Sozialität. Zur Phänomenologie sozialer Praxis. Sociologia Internationalis 41: 25-53.

Bourdieu, P., 1985: Sozialer Raum und ,Klassen'. Lecon sur la Lecon. Zwei Vorlesungen. Frankfurt am Main: Suhrkamp.

Bourdieu, P., 1987: Sozialer Sinn. Kritik der gesellschaftlichen Urteilskraft. Frankfurt am Main: Suhrkamp.

Bühl, W. L., 2002: Phänomenologische Soziologie. Ein kritischer Überblick. Konstanz: UVK.

Clarke, A. E., 2011: Von der Grounded-Theory-Methodologie zur Situationsanalyse. S. 207-229 in: G. Mey \& K. Mruck (Hrsg.), Grounded Theory Reader. Wiesbaden: VS.

Clarke, A. E., 2012: Situationsanalyse. Grounded Theory nach dem Postmodern Turn. Wiesbaden: VS.

Coenen, H., 1985a: Diesseits von subjektivem Sinn und kollektivem Zwang. Schütz, Durkheim, Merleau-Ponty: Phänomeno- logische Soziologie im Feld des zwischenleiblichen Verhaltens. München: Fink.

Coenen, H., 1985b: Leiblichkeit und Sozialität. Ein Grundproblem der phänomenologischen Soziologie. S.197-228 in: H. Petzold (Hrsg.), Leiblichkeit: Philosophische, gesellschaftliche und therapeutische Perspektiven. Paderborn: Junfermann.

Crossley, N., 1995: Merleau-Ponty, the Elusive Body and Carnal Sociology. Body \& Society 1: 43-64.

Crossley, N., 1996: Intersubjectivity. The Fabric of Social Becoming, London: Sage.

Dörpinghaus, S., 2013: Dem Gespür auf der Spur. Leibphänomenologische Studie zur Hebammenkunde am Beispiel der Unruhe. Freiburg/München: Alber.

Eberle, T. S., 2012: Phenomenology and Sociology: Divergent Interpretations of a Complex Relationship. S. 135-152 in: H. Nasu \& F. Chaput Waksler (Hrsg.), Interaction and Everyday Life. Phenomenological and Ethnomethodological Essays in Honor of George Psathas. Lanham: Lexington Books.

Eberlein, U., 2013: Leiberfahrung in kulturellen Praktiken. S. 89-110 in: W. Sohst (Hrsg.), Die Globalisierung der Affekte. Berlin: Xenomoi.

Ferguson, H., 2006: Phenomenological Sociology. Insight \& Experience in Modern Society. London: Sage.

Fischer, P., 2012: Phänomenologische Soziologie. Bielefeld: transcript.

Fischer, W., 2003: Körper und Zwischenleiblichkeit als Quelle und Produkt von Sozialität. Zeitschrift für qualitative Bildungs-, Beratungs- und Sozialforschung: 9-31.

Flam, H., 1990a: Emotional Man I: The Emotional Man and the Problem of Collective Action. International Sociology 5: 39-56.

Flam, H., 1990b: Emotional Man II: Corporate Actors as EmotionMotivated Emotion Managers. International Sociology 5: 225-234.

Goffman, E., 1971: Verhalten in sozialen Situationen. Strukturen und Regeln der Interaktion im öffentlichen Raum. Gütersloh: Bertelsmann.

Goffman, E., 1986: Interaktionsrituale. Über Verhalten in direkter Kommunikation. Frankfurt am Main: Suhrkamp.

Großheim, M., 2002: Erkennen oder Entscheiden. Der Begriff der „Situation“ zwischen theoretischer und praktischer Philosophie. Internationales Jahrbuch für Hermeneutik 1: 279-300.

Großheim, M., 2005: Der Situationsbegriff in der Philosophie. Mit einem Ausblick auf seine Anwendung in der Psychiatrie. S. 114-149 in: D. Schmoll \& A. Kuhlmann (Hrsg.), Symptom und Phänomen. Phänomenologische Zugänge zum kranken Menschen. Freiburg/München: Alber.

Großheim, M., S. Kluck \& H. Nörenberg, 2014: Kollektive Lebensgefühle. Zur Phänomenologie von Gemeinschaften. (Rostocker Phänomenologische Manuskripte 20). Rostock: Universität Rostock.

Gugutzer, R., 2002: Leib, Körper und Identität. Eine phänomenologisch-soziologische Untersuchung zur personalen Identität. Wiesbaden: Westdeutscher Verlag.

Gugutzer, R., 2004: Trendsport im Spannungsfeld von Körper, Selbst und Gesellschaft. Leib- und körpersoziologische Überlegungen. Sport und Gesellschaft 1: 219-243.

Gugutzer, R., 2005: Der Körper als Identitätsmedium: Essstörungen. S. 323-355 in: M. Schroer (Hrsg.), Soziologie des Körpers. Frankfurt am Main: Suhrkamp. 
Gugutzer, R., 2008: Leibliche Kommunikation im Tanz. S. 316-331 in: M. Großheim (Hrsg.), Neue Phänomenologie zwischen Praxis und Theorie. Festschrift für Hermann Schmitz. Freiburg/ München: Alber.

Gugutzer, R., 2010: Soziologie am Leitfaden des Leibes. Zur Neophänomenologie sozialen Handelns am Beispiel der Contact Improvisation. S. 165-184 in: F. Böhle \& M. Weihrich (Hrsg.), Die Körperlichkeit sozialen Handelns. Soziale Ordnung jenseits von Normen und Institutionen. Bielefeld: Transcript.

Gugutzer, R., 2012: Verkörperungen des Sozialen. Neophänomenologische Grundlagen und soziologische Analysen, Bielefeld: Transcript.

Gugutzer, R., 2014: Leibliche Praktiken der Geschlechterdifferenz. Eine neophänomenologische Kritik an der Körperpraxeologie in handlungstheoretischer Absicht. S. 91-106 in: C. Behnke, D. Lengersdorf \& S. Scholz (Hrsg.), Wissen - Methode Geschlecht: Erfassen des fraglos Gegebenen. Wiesbaden: VS.

Gugutzer, R., 2015a: Soziologie des Körpers (5. vollst. überarb. Aufl.) Bielefeld: Transcript.

Gugutzer, R., 2015b: Leibliche Interaktion mit Dingen, Sachen und Halbdingen. Zur Entgrenzung des Sozialen (nicht nur) im Sport. S. 105-122 in: H. K. Göbel \& S. Prinz (Hrsg.), Die Sinnlichkeit des Sozialen. Wahrnehmung und materielle Kultur. Bielefeld: Transcript.

Gugutzer, R., 2015c: Public Viewing. Ein sportiv gerahmtes kollektivleibliches Situationsritual. S. 71-96 in: R. Gugutzer \& M. Staack (Hrsg.), Körper und Ritual. Sozial- und kulturwissenschaftliche Zugänge und Analysen. Wiesbaden: VS.

Gugutzer, R., 2015d: (Neo-)Phänomenologie der Sportsucht. Theoretische und empirische Annäherungen. Sport und Gesellschaft 12: 189-214.

Gugutzer, R., 2016a: Self-tracking als Zeitgeist-Phänomen. S. 161-182 in: S. Duttweiler, R. Gugutzer, J.-H. Passoth \& J. Strübing (Hrsg.), Leben nach Zahlen. Self-tracking als Optimierungsprojekt? Bielefeld: Transcript.

Gugutzer, R., 2016b: Phänomenologie männlicher Leiberfahrung. S. 113-134 in: H. Landweer \& I. Marcinski (Hrsg.), Dem Erleben auf der Spur. Feminismus und die Philosophie des Leibes. Bielefeld: Transcript.

Gugutzer, R., 2017a: Leib und Körper als Erkenntnissubjekte. S. 381-394 in: R. Gugutzer, G. Klein \& M. Meuser (Hrsg.), Handbuch Körpersoziologie, Band 2: Forschungsfelder und Methodische Zugänge. Wiesbaden: VS.

Gugutzer, R., 2017b: Resonante Leiber, stumme Körper? Hartmut Rosas Resonanztheorie aus der Sicht einer verkörperten Soziologie. S. 69-85 in: C. H. Peters \& P. Schulze (Hrsg.), Resonanz in der Diskussion. Bielefeld: Transcript.

Gugutzer, R. \& N. Holterman, 2017: Der Dackelblick. Phänomenologie einer besonderen Hund-Mensch-Vergemeinschaftung. S. 265-284 in: R. Hitzler \& N. Burzan (Hrsg.), Auf den Hund gekommen. Interdisziplinäre Annäherung an ein Verhältnis. Wiesbaden: VS.

Gugutzer, R., G. Klein \& M. Meuser (Hrsg.), 2017: Handbuch Körpersoziologie (2 Bde.). Wiesbaden: VS.

Habermas, T., 1999: Geliebte Objekte. Symbole und Instrumente der Identitätsbildung. Frankfurt am Main: Suhrkamp.

Hasse, J., 2010: Raum der Performativität. „Augenblicksstätten“ im Situationsraum des Sozialen. Geographische Zeitschrift 98: 65-82.
Hitzler, R., 2005: Die Beschreibung der Struktur der Korrelate des Erlebens. Zum (möglichen) Stellenwert der Phänomenologie in der Soziologie. S. 230-240 in: U. Schimank \& R. Greshoff (Hrsg.), Was erklärt die Soziologie? Methodologien, Modelle, Perspektiven. Berlin: Lit.

Hitzler, R., 2008: Von der Lebenswelt zu den Erlebniswelten. Ein phänomenologischer Weg in soziologische Gegenwartsfragen S.131-140 in: J. Raab, M. Pfadenhauer, P. Stegmeier, J. Dreher \& B. Schnettler (Hrsg.), Phänomenologie und Soziologie. Theoretische Positionen, aktuelle Themenfelder, empirische Untersuchungen. Wiesbaden: VS.

Hitzler, R. \& A. Honer, 1984: Lebenswelt - Milieu - Situation. Terminologische Vorschläge zur theoretischen Verständigung. Kölner Zeitschrift für Soziologie und Sozialpsychologie 36: 56-74.

Hitzler, R. \& A. Honer, 1988: Der lebensweltliche Forschungsansatz. Neue Praxis 18: 496-501.

Hörning, K. H., 2004: Soziale Praxis zwischen Beharrung und Neuschöpfung. Ein Erkenntnis- und Theorieproblem. S.19-39 in: K. H. Hörning \& J. Reuter (Hrsg.), Doing Culture. Neue Positionen zum Verhältnis von Kultur und sozialer Praxis. Bielefeld: transcript.

Husserl, E., 1952: Ideen zu einer reinen Phänomenologie und phänomenologischen Philosophie II. Den Haag: Nijhoff.

Husserl, E., 1954: Die Krisis der europäischen Wissenschaften und die transzendentale Phänomenologie. Den Haag: Nijhoff.

Jäger, U., 2004: Der Körper, der Leib und die Soziologie. Entwurf einer Theorie der Inkorporierung, Königstein, Ts.: Helmer.

Knoblauch, H., 2009: Phänomenologische Soziologie. S. 299-322 in: G. Kneer \& M. Schroer (Hrsg.), Handbuch Soziologische Theorien. Wiesbaden: VS.

Landweer, H., 2016: Gemeinsame Gefühle und leibliche Resonanz. S. 137-174 in: U. Eberlein (Hrsg.), Zwischenleiblichkeit und bewegtes Verstehen. Intercorporeity, Movement and Tacit Knowledge. Bielefeld: transcript.

Lauterbach, Sr. M. J., 2014: „Gefühle mit der Autorität unbedingten Ernstes“. Eine Studie zur religiösen Erfahrung in Auseinandersetzung mit Jürgen Habermas und Hermann Schmitz. Freiburg/ München: Alber.

Latour, B., 2010: Eine neue Soziologie für eine neue Gesellschaft. Einführung in die Akteur-Netzwerk-Theorie. Frankfurt am Main: Suhrkamp.

Lindemann, G., 1992: Die leiblich-affektive Konstruktion des Geschlechts. Für eine Mikrosoziologie des Geschlechts unter der Haut. Zeitschrift für Soziologie 5: 330-346.

Lindemann, G., 1993: Das paradoxe Geschlecht. Transsexualität im Spannungsfeld von Körper, Leib und Gefühl, Frankfurt am Main: S. Fischer.

Lindemann, G., 1996: Zeichentheoretische Überlegungen zum Verhältnis von Körper und Leib. S. 146-175 in: A. Barkhaus, M. Mayer, N. Roughley \& D. Thürnau (Hrsg.), Identität, Leiblichkeit, Normativität. Neue Horizonte anthropologischen Denkens. Frankfurt am Main: Suhrkamp.

Lindemann, G., 2005: Die Verkörperung des Sozialen. Theoriekonstruktionen und empirische Forschungsperspektiven. S. 114-138 in: M. Schroer (Hrsg.), Soziologie des Körpers. Frankfurt am Main: Suhrkamp.

Lindemann, G., 2014: Weltzugänge. Die mehrdimensionale Ordnung des Sozialen. Weilerswirst: Velbrück. 
Lindemann, G., 2017: Leiblichkeit und Körper. S. 57-66 in: R. Gugutzer, G. Klein \& M. Meuser (Hrsg.), Handbuch Körpersoziologie, Bd.1: Grundbegriffe und theoretische Perspektiven. Wiesbaden: Springer VS.

Luckmann, T., 1979: Phänomenologie und Soziologie. S. 196-205 in: W. M. Sprondel \& R. Grathoff (Hrsg.), Alfred Schütz und die Idee des Alltags in den Sozialwissenschaften. Stuttgart: Enke.

Luckmann, T., 1992: Theorie des sozialen Handelns. Berlin/New York: de Gruyter.

Luckmann, T., 2008: Konstitution, Konstruktion. Phänomenologie, Sozialwissenschaft. S. 33-40 in: J. Raab, M. Pfadenhauer, P. Stegmeier, J. Dreher \& B. Schnettler (Hrsg.), Phänomenologie und Soziologie. Theoretische Positionen, aktuelle Themenfelder, empirische Untersuchungen. Wiesbaden: VS.

Lüdtke, N., 2008: Intersubjektivität bei Schütz - oder: Ist die Frage nach dem Anderen aus der Phänomenologie entlassen? S. 187-197 in: J. Raab, M. Pfadenhauer, P. Stegmeier, J. Dreher \& B. Schnettler (Hrsg.), Phänomenologie und Soziologie. Theoretische Positionen, aktuelle Themenfelder, empirische Untersuchungen. Wiesbaden: VS.

Merleau-Ponty, M., 1994: Das Sichtbare und das Unsichtbare: gefolgt von Arbeitsnotizen. München: Fink.

Meyer-Drawe, K., 1984: Leiblichkeit und Sozialität. Phänomenologische Beiträge zu einer pädagogischen Theorie der Intersubjektivität. München: Fink.

Nasu, H., 2012: The Phenomenological Sociology Movement in the United States: The Developmental Process of an Intellectual Movement. S. 3-21 in: H. Nasu \& F. C. Waksler (Hrsg.), Interaction and Everyday Life. Phenomenological and Ethnomethodological Essays in Honor of George Psathas. Lanham: Lexington Books.

Plessner, H., 1975: Die Stufen des Organischen und der Mensch, Berlin/New York: de Gruyter.

Raab, J., M. Pfadenhauer, P. Stegmeier, J. Dreher \& B. Schnettler (Hrsg.), 2008: Phänomenologie und Soziologie. Theoretische Positionen, aktuelle Problemfelder und empirische Umsetzungen. Wiesbaden: VS.

Rosa, H., 2016: Resonanz. Eine Soziologie der Weltbeziehung. Berlin: Suhrkamp.

Sartre, J.-P., 1993: Das Sein und das Nichts. Versuch einer phänomenologischen Ontologie. Reinbek: Rowohlt.

Schimank, U., 2000: Handeln und Strukturen. Einführung in die akteurtheoretische Soziologie. Weinheim/München: Juventa.

Schmid, W., 1999: Philosophie der Lebenskunst. Eine Grundlegung. Frankfurt am Main: Suhrkamp.

Schmidt-Degenhard, M., 2005: Interpretative Situageneseforschung. S. 150-165 in: D. Schmoll \& A. Kuhlmann (Hrsg.), Symptom und Phänomen. Phänomenologische Zugänge zum kranken Menschen. Freiburg/München: Alber.

Schmitz, H., 1964: System der Philosophie, Band 1: Die Gegenwart. Bonn: Bouvier.

Schmitz, H., 1965: System der Philosophie. Band 2, Teil 1: Der Leib. Bonn: Bouvier.

Schmitz, H., 1966: System der Philosophie. Band 2, Teil 2: Der Leib im Spiegel der Kunst. Bonn: Bouvier.

Schmitz, H., 1967: System der Philosophie, Band 3, Teil 1: Der leibliche Raum. Bonn: Bouvier.

Schmitz, H., 1969: System der Philosophie, Band 3, Teil 2: Der Gefühlsraum. Bonn: Bouvier.
Schmitz, H., 1973: System der Philosophie, Band 3, Teil 3: Der Rechtsraum. Bonn: Bouvier.

Schmitz, H., 1977: System der Philosophie, Band 3, Teil 4: Das Göttliche und der Raum. Bonn: Bouvier.

Schmitz, H., 1978: System der Philosophie, Band 3, Teil 5: Die Wahrnehmung. Bonn: Bouvier.

Schmitz, H., 1980a: System der Philosophie, Band 4: Die Person. Bonn: Bouvier.

Schmitz, H., 1980b: System der Philosophie, Band 5: Die Aufhebung der Gegenwart. Bonn: Bouvier.

Schmitz, H., 1980c: Neue Phänomenologie. Bonn: Bouvier.

Schmitz, H., 1990: Der unerschöpfliche Gegenstand. Grundzüge der Philosophie. Bonn: Bouvier.

Schmitz, H., 1999: Adolf Hitler in der Geschichte. Bonn: Bouvier.

Schmitz, H., 2002: Was ist ein Phänomen? S. 13-22 in: H. Schmitz, G. Marx \& A. Moldzio (Hrsg.), Begriffene Erfahrung. Beiträge zur antireduktionistischen Phänomenologie. Rostock: Koch.

Schmitz, H., 2003: Was ist Neue Phänomenologie? Rostock: Koch.

Schmitz, H., 2005: Situationen und Konstellationen. Wider die Ideologie der Vernetzung. Freiburg/München: Alber.

Schmitz, H., 2009: Kurze Einführung in die Neue Phänomenologie. Freiburg/München: Alber.

Schmitz, H., 2011: Der Leib. Berlin/Boston: de Gruyter.

Schmitz, H., 2012: Im Reich der Normen. Freiburg/München: Alber.

Schmitz, H., 2014: Atmosphären. Freiburg/München: Alber.

Schmitz, H., 2016: Ausgrabungen zum wirklichen Leben. Eine Bilanz. Freiburg/München: Alber.

Schütz, A., 1960: Der sinnhafte Aufbau der sozialen Welt. Eine Einleitung in die verstehende Soziologie. Frankfurt am Main: Suhrkamp.

Schütz, A., 1971a: Gesammelte Aufsätze I: Das Problem der Wirklichkeit. Den Haag: Nijhoff.

Schütz, A., 1971b: Gesammelte Aufsätze III: Studien zur phänomenologischen Philosophie. Den Haag: Nijhoff.

Schütz, A., 1971c: Phänomenologie und die Sozialwissenschaften. S. 136-161 in: A. Schütz, Gesammelte Aufsätze I: Das Problem der Wirklichkeit. Den Haag: Nijhoff.

Schütz, A. \& T. Luckmann, 2003: Strukturen der Lebenswelt. Konstanz: UVK.

Schützeichel, R., 2016: Zur Soziologie des Rechtsgefühls. S. 65-99 in: H. Landweer \& D. Koppelberg (Hrsg.), Recht und Emotion I: Verkannte Zusammenhänge. Freiburg/München: Alber.

Simmel, G., 1992: Exkurs über die Soziologie der Sinne. S. 722-742 in: G. Simmel, Soziologie. Untersuchungen über die Formen der Vergesellschaftung. Frankfurt am Main: Suhrkamp.

Soentgen, J., 1998: Die verdeckte Wirklichkeit. Einführung in die Neue Phänomenologie von Hermann Schmitz. Bonn: Bouvier.

Sohst, H. \& H. Schmitz, 2005: Hermann Schmitz im Dialog. Neun neugierige und kritische Fragen an die Neue Phänomenologie. Berlin: Xenomoi.

Srubar, I., 2007: Phänomenologie und soziologische Theorie. Aufsätze zur pragmatischen Lebenswelttheorie. Wiesbaden: VS.

Suber, D., 2007: Die soziologische Kritik der philosophischen Vernunft. Zum Verhältnis von Soziologie und Philosophie um 1900. Bielefeld: Transcript.

Taylor, C., 1986: Leibliches Handeln. S. 194-217 in: A. Métraux \& B. Waldenfels (Hrsg.), Leibhaftige Vernunft. Spuren von MerleauPontys Denken. München: Fink. 
Uzarewicz, M., 2011: Der Leib und die Grenzen der Gesellschaft. Eine neophänomenologische Soziologie des Transhumanen. Stuttgart: Lucius \& Lucius.

Waldenfels, B., 2015: Sozialität und Alterität. Modi sozialer Erfahrung. Berlin: Suhrkamp.

Young, I. M., 1993: Werfen wie ein Mädchen. Eine Phänomenologie weiblichen Körperverhaltens, weiblicher Motilität und Räumlichkeit. Deutsche Zeitschrift für Philosophie 41: 707-723.

Ziemann, A. (Hrsg.), 2013a: Offene Ordnung? Philosophie und Soziologie der Situation. Wiesbaden: VS.

Ziemann, A., 2013b: Zur Philosophie und Soziologie der Situation - eine Einführung. S. 7-18 in: A. Ziemann (Hrsg.), Offene Ordnung? Philosophie und Soziologie der Situation. Wiesbaden: Springer VS.

Ziemann, A., 2013c: Soziologische Strukturlogiken der Situation. S. 105-129 in: A. Ziemann (Hrsg.), Offene Ordnung? Philosophie und Soziologie der Situation. Wiesbaden: Springer VS.

\section{Autorenvorstellung}

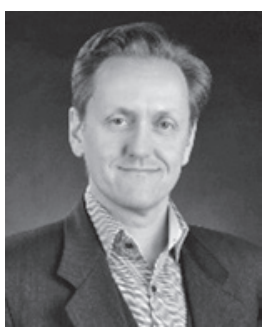

\section{Robert Gugutzer}

Goethe-Universität Frankfurt a. M., Institut für Sportwissenschaften, Abt. Sozialwissenschaften des Sports, Ginnheimer Landstraße 39, 60487 Frankfurt a. M. Tel.: 069/798-245 29, Fax: 069/798-24539, E-mail: gugutzer@sport.uni-frankfurt.de

Robert Gugutzer, geb. 1967 in Dorfen. Studium der Soziologie, Psychologie und Politikwissenschaft in Tübingen und München. Promotion 2001 an der Universität Halle-Wittenberg, Habilitation 2011 an der Universität Augsburg. Von 1999-2002 wissenschaftlicher Mitarbeiter im SFB „Reflexive Modernisierung“ an der Universität Augsburg, von 2001-2009 wissenschaftlicher Mitarbeiter an der TU München, seit Oktober 2009 Professor für Sozialwissenschaften des Sports an der Goethe-Universität Frankfurt am Main. Forschungsschwerpunkte: Körpersoziologie, Sportsoziologie, Filmsoziologie, Religionssoziologie, Leibphänomenologie, Sozialtheorie Wichtigste Buchpublikationen: Handbuch Körpersoziologie, 2 Bde. (hrsg. mit G. Klein \& M. Meuser), Wiesbaden 2017; Soziologie des Körpers (5. Aufl.), Bielefeld 2015; Verkörperungen des Sozialen. Neophänomenologische Grundlagen und soziologische Analysen, Bielefeld 2012; body turn. Perspektiven der Soziologie des Körpers und des Sports (als Hrsg.), Bielefeld 2006; Leib, Körper und Identität. Eine phänomenologisch-soziologische Untersuchung zur personalen Identität, Wiesbaden 2002. 